\title{
D0P-2 D2-Like Receptor Regulates UNC-7 Innexins to Attenuate Recurrent Sensory Motor Neurons during C. elegans Copulation
}

\author{
Paola A. Correa, ${ }^{1}$ Todd Gruninger, ${ }^{2}$ and L. René García ${ }^{1,3}$ \\ ${ }^{1}$ Department of Biology, Texas A\&M University, College Station, Texas 77843, 2Jesuit College Preparatory, Dallas, Texas 75244, and ${ }^{3}$ Howard Hughes \\ Medical Institute
}

\begin{abstract}
Neuromodulation of self-amplifying circuits directs context-dependent behavioral executions. Although recurrent networks are found throughout the Caenorhabditis elegans connectome, few reports describe the mechanisms that regulate reciprocal neural activity during complex behavior. We used $C$. elegans male copulation to dissect how a goal-oriented motor behavior is regulated by recurrently wired sensory-motor neurons. As the male tail presses against the hermaphrodite's vulva, cholinergic and glutamatergic reciprocal innervations of post cloaca sensilla (PCS) neurons (PCA, PCB, and PCC), hook neurons (HOA, HOB), and their postsynaptic sex muscles execute rhythmic copulatory spicule thrusts. These repetitive spicule movements continue until the male shifts off the vulva or genital penetration is accomplished. However, the signaling mechanism that temporally and spatially restricts repetitive intromission attempts to vulva cues was unclear. Here, we report that confinement of spicule insertion attempts to the vulva is facilitated by D2-like receptor modulation of gap-junctions between PCB and the hook sensillum. We isolated a missense mutation in the UNC-7(L) gap-junction isoform, which perturbs DOP-2 signaling in the PCB neuron and its electrical partner, HOA. The glutamate-gated chloride channel AVR-14 is expressed in HOA. Our analysis of the unc-7 mutant allele indicates that when DOP-2 promotes UNC-7 electrical communication, AVR-14-mediated inhibitory signals pass from HOA to PCB. As a consequence, PCB is less receptive to be stimulated by its recurrent synaptic partner, PCA. Behavioral observations suggest that dopamine neuromodulation of UNC-7 ensures attenuation of recursive intromission attempts when the male disengages or is dislodged from the hermaphrodite genitalia.
\end{abstract}

Key words: copulation; D2-like receptors; dopamine signaling; gap-junctions; goal-oriented behavior; recurrent neuronal network

Significance Statement

Using C. elegans male copulation as a model, we found that the neurotransmitter dopamine stimulates D2-like receptors in two sensory circuits to terminate futile behavioral loops. The D2-like receptors promote inhibitory electrical junction activity between a chemosensory and a mechanosensory circuit. Therefore, both systems are attenuated and the animal ceases the recursive behavior.

\section{Introduction}

The mammalian CNS facilitates context-dependent behaviors through recurrent neuronal network (RNN) modulation

\footnotetext{
Received March 10, 2015; revised May 22, 2015; accepted June 8, 2015.

Author contributions: P.A.C. and T.G. designed research; P.A.C. and T.G. performed research; P.A.C. and L.R.G. analyzed data; P.A.C. and L.R.G. wrote the paper.

This work was supported by the Howard Hughes Medical Institute. We thank the Caenorhabditis Genetic Center, which provided some strains, which is funded by National Institutes of Health Office of Research Infrastructure Programs (P40 0D010440). We thank all the members of the L.R.G. laboratory; Dr. Guo and Dr. Leboeuf for critical reading of the manuscript; Dr. Gualberto for technical help; and the Caenorhabditis Genetic Center for strains.

The authors declare no competing financial interests.

Correspondence should be addressed to Dr. L. René García, Department of Biology, Texas A\&M University, 3258 TAMU, College Station, TX 77843-3258. E-mail: rgarcia@mail.bio.tamu.edu.

DOI:10.1523/JNEUROSCI.0940-15.2015

Copyright $\odot 2015$ the authors $\quad 0270-6474 / 15 / 359990-15 \$ 15.00 / 0$
}

(Destexhe and Contreras, 2006). As the CNS receives environmental signals, dopamine (DA) midbrain secretion integrates incoming information and promotes the appropriate behaviors (Redgrave and Gurney, 2006; Shao et al., 2013). DA neurons project into RNNs in cortical regions (Brozoski et al., 1979; Goldman-Rakic et al., 1989) to adjust cognitive tasks via D1- or D2-like receptors (Matsumoto and Takada, 2013; Haber, 2014). In primates, D1-like signaling amplifies prefrontal and occipital cortical RNNs to promote purposeful eye movements to visual targets (Noudoost and Moore, 2011; Zaldivar et al., 2014). In contrast, how D2-like signaling alters $\mathrm{RNN}^{\prime}$ 's control on behavior remains unknown because the complex cortical connectivity presents a challenge for behavioral studies. In this regard, the connectome, molecular tools, 
and genetics make Caenorhabditis elegans tractable to dissect how neural circuits regulate purposeful behaviors.

Published studies have examined how DA modulates C. elegans locomotion in different environments. When the worm encounters food, eight mechanosensory DA neurons alter crawling speed or directionality via D1-like and D2-like receptors. The D1-like pathway enhances nociceptive neuron excitability. This change in cell activity mediates how food affects chemicalrepellant sensitization and promotes escape responses (Sulston et al., 1975; Sawin et al., 2000; Sugiura et al., 2005; Ezcurra et al., 2011). The opposing D2-like cascade dampens cholinergic ventral cord excitability to decrease the animal's velocity after entering food (Sawin et al., 2000; Chase et al., 2004; Allen et al., 2011). These observations suggest parallels with vertebrate DA signaling mechanisms; however, these studies focused on DA's role in regulating behavioral states. Here, we demonstrate that $C$. elegans male mating is suitable for also studying how DA regulates recursive context-dependent behaviors.

Reciprocally connected sensory-motor neurons in the C. elegans' male tail suggest that recurrent networks control most steps of copulation, including spicule intromission behavior (Jarrell et al., 2012). The 18 sensory ray neuron pairs (termed R1A/B through $\mathrm{R} 9 \mathrm{~A} / \mathrm{B}$ ) induce male tail contact with the hermaphrodite (Koo et al., 2011) and promote backward locomotion as he searches for the vulva (Sherlekar et al., 2013). When he approaches the vulva, the hook sensillum neurons HOA and $\mathrm{HOB}$ sense long-range vulva cues (Liu and Sternberg, 1995; Barr and Sternberg, 1999) and subsequently reduce the male's velocity, allowing the post cloacal sensilla (PCS) to detect short-range vulva features (Liu and Sternberg, 1995; Barr and Sternberg, 1999).

The bilateral PCS consist of the reciprocally connected PCA, PCB, and PCC sensory-motor neurons (see Fig. 1). They stabilize the male over the vulva while inducing rhythmic spicule thrusts. The cholinergic PCB, PCC (Garcia et al., 2001), and glutamatergic PCA pairs (LeBoeuf et al., 2014) stimulate posterior sex muscle contractions. Electrical coupling of these sex muscles with the spicule protractor muscles causes simultaneous muscle group contraction, leading to rhythmic spicule thrusts. This repetitive spicule "prodding" lasts until full penetration or if the male shifts off the vulva (Liu et al., 2011).

We reported that cholinergic and DA signaling stimulates acetylcholine and D2-like receptors to regulate rhythmic spicule activity. DA neurotransmission from R5A, R7A, and R9A ray neurons dampens persistent cholinergic-mediated intromission attempts, causing the male to terminate prodding after prolonged attempts, or if he shifts off the vulva (Sulston et al., 1975; Correa et al., 2012). Because these DA rays chemically synapse onto HOA, PCA, PCB, and the sex muscles (see Figure 1), DA likely down-modulates these components.

Here, we report that restricting repetitive behaviors to the vulva requires D2-like modulation of gap-junctions between the PCS and hook sensillum. We isolated an unc-7 gap-junction mutation that disrupts DA signaling in the PCB and HOA neurons, suggesting that the gap-junction is a D2-like signaling effector. Our analyses indicate that the DOP-2 D2-like receptor promotes UNC-7 electrical communication to increase inhibitory glutamate-induced signals from HOA into PCB. This mechanism diminishes the PCS interneuronal stimulation to ensure termination of spicule intromission attempts, when the male moves off the vulva.

\section{Materials and Methods}

Strains and culture methods. Strains were maintained at $20^{\circ} \mathrm{C}$ on NGM agar plates and fed with Escherichia coli OP50. The avr-14(ad1302) LGI, avr-15(ad1051) LGV (Dent et al., 1997), and avr-14(ad1302) (Dent et al., 2000); avr-15(ad1051) L4 hermaphrodites were heat shocked at $30^{\circ} \mathrm{C}$ to obtain males carrying these alleles. Assays performed with these heatshocked mutant males were conducted in parallel with N2 heat-shocked males. Because the unc-7(hs9) and unc-7(hs10)LGX are temperaturesensitive alleles displaying a locomotion defect at $15^{\circ} \mathrm{C}$ (Hecht et al., 1996), we crossed him- 5 males onto these hermaphrodites at $20^{\circ} \mathrm{C}$ for $5-8 \mathrm{~h}$ and then placed the mating plates at $15^{\circ} \mathrm{C}$ until the $\mathrm{F} 1$ males became adults. When these males were used for drug test, we also grew him- 5 males at $15^{\circ} \mathrm{C}$ as a control. Similarly, we obtained males from the unc-7(e139) LGX (Brenner, 1974) by crossing with him-5 males to unc7(e139) hermaphrodites. Otherwise, all C. elegans males contain the him5(e1490) allele LGV (Hodgkin et al., 1979). Additional alleles used were as follows: unc-7(e5) LGX (Brenner, 1974), dop-2(vs105) LGV (Chase et al., 2004), pha-1 (e2123) LGIII (Schnabel and Schnabel, 1990), lite1(ce314) LGX (Edwards et al., 2008), $g l c-1$ (pk54) LGV(Vassilatis et al., 1997), and unc-9(e101)LGX (Brenner, 1974).

The unc-7(rg396) allele was isolated from an ethyl methanesulfonate (EMS) mutagenesis screen that selected for males displaying constitutively protracted spicules (Prc phenotype). In brief, the (rg1) mutation in lev-11/tropomyosin suppresses the Prc phenotype of the unc-103(sy557)/ ERG $\mathrm{K}^{+}$channel allele. The $r g 1$ allele causes the pharyngeal feeding circuit to reduce the male's sexual libido, thus indirectly suppressing the unc-103(sy557)-induced Prc phenotype (Gruninger et al., 2008). The unc-103; lev-11 strain was used for the EMS mutagenesis to identify extragenic mutations that restored the Prc phenotype. The pseudorevertants were then pharmacologically tested for their DA + arecoline (ARE) response. The rg396 strain was identified and outcrossed five times to repulse $u n c-103$ and $l e v-11$. The allele was mapped to LGX using classical genetic two- and three-point mapping analysis. Whole genome sequencing was conducted by BGI Americas Corporation (http://www. genomics.cn/en), and the rg396 allele was found to be located in the unc-7 locus. The rg396 mutation changed the wild-type sequence AAACTGCGCTCAG to the mutant sequence AAACTACGCTCAG.

Transgenic strains include the following: pha-1(lf), lite-1(lf), rgEx625 [Punc-17:ChR2::YFP, Peat-4: GCaMP6::SL2:::mDsRed, pha-1(+)]; pha1(lf), lite-1(lf), rgEx601 [Punc-17: GCaMP6::SL2:::mDsRed, Peat-4: ChR2::YFP, pha-1(+)]; pha-1(lf), lite-1(lf) dop-2(lf), rgEx601 [Punc-17:GCaMP6::SL2:::mDsRed, Peat-4:ChR2::YFP, pha-1(+)]; pha1(lf), unc-7(rg396), rgEx601 [Punc-17: GCaMP6::SL2:::mDsRed, Peat-4:ChR2::YFP, pha-1(+)]; pha-1(lf), lite-1(lf), rgEx431 [Phsp-16:egl2(n693gf)cDNA; Punc-103E:mDsRed; pha-1(+)]; pha-1(lf), unc-7(e5), rgEx653 [Punc-7(5kb):unc-7::YFP + coinjected SL2::GFP fragment; pha1(+)]; pha-1(lf), rgEx652 [Punc-7: unc-7(A59T)::YFP + coinjected SL2::GFP fragment; pha-1(+)]; pha-1(lf), rgEx653 [Punc-7(5kb): unc-7::YFP + coinjected SL2::GFP fragment; pha-1(+)]; pha-1(lf), rgEx668 [Punc-7: unc-7 (A59T)::YFP; + coinjected SL2::GFP fragment; pha-1(+)]; pha-1(lf), unc-7 (rg396), rgEx644 [Punc-7: unc-7::YFP; pha1(+)]; pha-1(lf), rgEx672 [Plev-11:unc-7(A59T)::YFP; pha-1(+)]; pha1(lf), rgEx673 [Paex-3:unc-7 (A59T)::YFP; pha-1(+)]; pha-1(lf), rgEx671 [Punc-103E: unc-7 (A59T)::YFP; pha-1(+)]; pha-1(lf) rgEx674 [Pdop-2: unc-7 (A59T)::YFP; pha-1(+)]; pha-1(lf) rgEx649 [Pdop-2: unc-7::YFP; pha-1(+)]; pha-1(lf) rgEx [Punc-7(7kb): unc-7::YFP coinjected SL2::GFP fragment; pha-1(+)]; pha-1(lf) rgEx734 [Peat-4: unc-7(A59T)::YFP; pha-1(+)]; pha-1(lf) rgEx733 [Pavr-14: unc-7 (A59T)::YFP; pha-1(+)]; pha-1(lf) rgEx736 [Peat-4: unc-7::YFP; pha-1(+)]; pha-1(lf) rgEx735 [Pavr-14: unc-7::YFP; pha-1(+)]; pha-1(lf), lite-1(lf), dop-2(lf), rgEx625 [Punc-17:ChR2::YFP, Peat-4: GCaMP6::SL2:::mDsRed, pha-1(+)].

Plasmids: reporters of unc-7 and avr-14 expression. Primer sequences are provided in Table 1. A $5 \mathrm{~kb}$ region upstream of the avr-14 ATG and the first 7 codons was PCR-amplified using the primers ATTB1Pavr-14F and ATTB2Pavr14R. A $5.7 \mathrm{~kb}$ and a $7.7 \mathrm{~kb}$ region upstream of the unc-7 ATG was PCR-amplified using the primers attb1Punc7F, Punc7 (8kb)F, and Punc7(8/9)R. All these primers contained Gateway ATTB sites, which allowed one avr-14 and two unc-7 PCR products to be recombined 


\section{Table 1. Primers used in this study}

\begin{tabular}{ll}
\hline Primer name & Sequence \\
\hline attb1Pavr-14 forward & ggggacaagtttgtacaaaaaagcaggctcc ctgccccgagaacctaaacaacattgaat \\
attb1Pavr-14 reverse & ggg gac cac ttt gta caa gaa agc tgg gta cgtcagtcgataatgccacatctgaaagt \\
Punc7(8 kb) forward & ggggacaagtttgtacaaaaaagcaggctcc tatcgcaagttcacctcatctgtgccttc \\
Punc7(8/9) reverse & ggg gac cac ttt gta caa gaa agc tgg gta acatccgtctaaacttgcacgttgaggcg \\
attb1Punc7 forward & ggggacaagtttgtacaaaaaagcaggctcc cccatagagagtgatgggcccaaccttc \\
UNC-7(gene)attb1 & ggggacaagtttgtacaaaaaagcaggctcc atgctcggctcctccagcaatcct \\
UNC-7(gene)attb2 & ggg gac cac ttt gta caa gaa agc tgg gta cggtctatcgtcccttgaccgtgttc \\
\hline
\end{tabular}

using BP clonase (Invitrogen), into the low copy Gateway entry vector pDG15, to generate pPC112, pPC76, and pPC103, respectively. pDG15 contains the p15A origin of replication, which has a copy number of 15 per cell. The vector allows $E$. coli to harbor cloned-unstable sequences better than plasmids containing the ColE1 origin of replication (Reiner et al., 2006). To place the avr-14 and unc-7 promoter sequences upstream of YFP, the pPC112, pPC76, and pPC103 vectors were individually recombined with the YFP destination vector pGW322YFP (Reiner et al., 2006) (a plasmid containing the gateway destination ATTR Reading frame Cassette C.1 upstream of YFP) using LR clonase (Invitrogen) to make plasmids pPC111, pPC78, and pPC101, respectively.

Expression of unc-7 and cell-specific expression of unc-7(A59T). A $5.7 \mathrm{~kb}$ genomic unc-7-containing sequence from the ATG to the penultimate codon was PCR-amplified via primers UNC7(gene)Attbland UNC7(gene)Attb2 (Table 1). Because these PCR primers contained Gateway ATTB sites, unc-7(genomic DNA) was recombined using BP clonase into pDG15, to generate pPC86. To make pPC87 [unc-7::YFP], pPC86 was recombined with pGW322YFP using LR clonase. To drive unc-7::YFP expression from different promoters, pPC87 was cut with AfeIII and ligated with the Gateway Vector Conversion Reading frame Cassette B (Invitrogen) to generate the destination vector pPC89. The LR sites flanking $u n c-7$ were removed using single site mutagenesis to obtain the pPC89R plasmid. To drive unc-7 (genomic):YFP expression from unc-7(5kb) and unc-7(7.7) endogenous promoters, the plasmids pPC86 and pPC103 were individually recombined into PPC89R using LR clonase to obtain pPC90 and pPC102. Moreover, the A59T mutation in the first $u n c-7$ exon was introduced into PPC89R via single site mutagenesis to generate pPC99. To drive unc-7(A59T):YFP expression from the unc$7(5 \mathrm{~kb})$ promoter, the pPC86 plasmid was recombined into pPC99 to obtain pPC100.

To generate the minigene $u n c-7$ and $u n c-7(\mathrm{~A} 59 \mathrm{~T})$ versions, the following changes were made using single site mutagenesis: two large intronic regions (intron 2 and 5; see Fig. $3 C$ ) were removed from the pPC89R plasmid to generate pPC93 and pPC94, respectively. Subsequently, the A59T mutation was introduced into pPC94 to generate pPC104. To drive unc-7(minigene)::YFP expression from the dop-2 promoter (Pdop-2), the glutamatergic neuronal promoter (Peat-4), and the glutamate chloride channel promoter (Pavr-14), the plasmids pPC1 (Correa et al., 2012), pPC52, and pPC112 were individually recombined into pPC94 using LR clonase, to generate pPC95 [Pdop-2:unc-7::YFP], pPC121 [Peat-4: unc-7::YFP], and pPC123[Pavr-14: unc-7::YFP].

To drive unc-7(A59Tminigene)::YFP expression from the dop-2 promoter (Pdop-2), a sex-muscle-expressing promoter (Punc-103E), a pan-muscular promoter (Plev-11), the pan-neuronal promoter (Paex-3), the glutamatergic neuronal promoter (Peat-4), and the glutamate chloride channel promoter (Pavr-14), the plasmids pPC1 (Correa et al., 2012), pLR21 (Reiner et al., 2006), pLR22 (Gruninger et al., 2008), pLR35 (LeBoeuf et al., 2007), pPC52, and pPC112 were individually recombined into pPC104 using LR clonase, to make pPC108 [Pdop-2:unc-7(A59T)::YFP], pPC105 [Punc103E: unc-7 $(A 59 T):: Y F P], \quad$ pPC106 [Plev11: unc-7(A59T)::YFP], pPC107 [Paex-3: unc-7(A59T)::YFP], pPC113 [Peat-4: unc-7(A59T)::YFP], and pPC115 [Pavr-14: unc-7(A59T)::YFP].

GCaMP6 and ChR2 plasmids. We inserted an SL2-accepting transsplice site followed by the mDsRed gene and an unc-54 3'UTR immediately downstream of Gateway ATTR Reading frame Cassette C.1and GCaMP6(M) to create the vector pLR305. In the pLR167 (Liu et al.,
2011) plasmid containing the ChR2:YFP (no introns) downstream of Gateway ATTR Reading frame Cassette C.1, the YFP was swapped for the YFP containing all introns from the pGWYFP plasmid to create pBL248 for better fluorescence expression. This was done through In-fusion (Clonetech). To introduce promoters upstream of the GCaMP6 and DsRed sequences or ChR2:YFP, the plasmids containing the promoters Punc-17(pBL228) and Peat-4(pPC52) (LeBoeuf et al., 2014) were individually recombined with pLR305 and pBL248 to generate the plasmids: pPC64 [Punc-17:GCaMP6::SL2:::mDsRed], pBL250 [Punc-17: ChR2::YFP], pPC68 [Peat4: GCaMP6::SL2:::mDsRed], and pPC54 [Peat-4:ChR2::YFP].

Transgenics. To generate transmitting lines expressing the respective plasmids, pBX1 was coinjected $(50-100 \mathrm{ng} / \mu \mathrm{l})$ into strains that contained the pha-1(e2123) allele. Transgenic lines that could stably propagate at $20^{\circ} \mathrm{C}$ were kept for further analysis. For all injections, pUC18 was used to make the final DNA concentration $200 \mathrm{ng} / \mu \mathrm{l}$. The expression constructs pPC111, pPC76, and pPC101 were injected at $80 \mathrm{ng} / \mu \mathrm{l}$ into pha-, him-5, lite- 1 hermaphrodites. To further analyze the unc-7 expression, the pPC90 $(30 \mathrm{ng} / \mu \mathrm{l})$ and $\mathrm{pPC} 102(50 \mathrm{ng} / \mu \mathrm{l})$ were coinjected with $25 \mathrm{ng} / \mu \mathrm{l}$ of PJP1 (SL2:GFP) into pha-1 hermaphrodites.

To rescue the $u n c-7(r g 396)$ and $u n c-7(e 5)$ DA drug response, pPC90 was injected $(80 \mathrm{ng} / \mu \mathrm{l})$ into pha-1, unc-7(rg396), and pha-1, unc-7(e5) hermaphrodites, respectively. To phenocopy the unc-7(rg396) DA + ARE drug resistance, we injected pPC100 (10 ng/ $\mu$ l) into pha-1 hermaphrodites. Additionally, to phenocopy the unc-7( $r$ 396) phenotype in specific tissues, the pPC105, pPC106, pPC107, pPC108, pPC113, and pPC115 plasmids $(50 \mathrm{ng} / \mu \mathrm{l})$ were individually injected into pha-1 hermaphrodites. To control some of the phenocopy experiments, pPC 121 and pPC123 (50 ng/ $/$ l) were individually injected into pha-1 hermaphrodites.

To label separately the cholinergic and glutamatergic male PCS neurons, either with GCaMP6 or ChR2:YFP, pha-1, him-5, lite-1 hermaphrodites were injected with pPC64 [Punc-17:GCaMP6::SL2:::mDsRed] (60 ng/ $\mu \mathrm{l})$, pPC54 [Punc-17:ChR2::YFP] (70 ng/ $\mu \mathrm{l})$, or pBL250 [Punc-17:ChR2::YFP] $(60 \mathrm{ng} / \mu \mathrm{l})$ and pPC68[Peat4: GCaMP6M::SL2:::mDsRed] $(60 \mathrm{ng} / \mu \mathrm{l})$.

Behavioral assays and drug test. We isolated virgin males from noncrowded plates at early or mid-L4 stage for behavioral and pharmacology assays. They were kept in groups of 10-20 animals. On the assay day, all males with constitutive spicule protraction were discarded before any further behavioral or drug test analysis.

To assay agonist-induced spicule protraction, we dissolved ARE (Acrose Organics) and DA (Sigma) in water to make a stock solution of 100 $\mathrm{mm}$ and $50 \mathrm{~mm}$, respectively. Abamectin (ABA) and ivermectin (IVR) were dissolved in DMSO to make stock solutions of both drugs at 10 $\mathrm{mg} / \mathrm{ml}$. We added $400 \mu \mathrm{l}$ of either drug combinations to a three well round-bottom Pyrex titer dish. Five to 10 males were then transferred to the drug bath. All drug tests scored the number of males that protracted their spicules by directly observing spicule protraction at $20^{\circ} \mathrm{C}$ for at least $10 \mathrm{~s}$, in a 5 min observation window. For DA and ARE exposure, these drugs were premixed. Because ABA and IVR slowly induce detectable behavioral responses, such as locomotor paralysis, worms were bathed in avermectins for $7 \mathrm{~min}$ and then ARE was added at a small volume, such that the final ABA or IVR concentration was not significantly changed. For any of these drug mixtures, ARE concentration was set at the $\mathrm{EC}_{90}$ concentration (1 mM).

To score multiple mating behavioral parameters for individual males, we digitally recorded every mating event from the time a male contacted a hermaphrodite with the ventral side of its tail, until spicule insertion or $5 \mathrm{~min}$. To ensure consistent behavioral measurements, we recorded and watched mating movies for mutants or transgenic males and control groups on the same date. For mating behavioral assays, 10-15 heatshocked paralyzed pha-1, lite-1 rgEx431 [Phsp-16:egl-2(n693gf)cDNA; Punc-103E:mDsRed; pha-1(+)] hermaphrodites were evenly spaced on the lawn. A virgin 1-day old male was then placed in the middle of the hermaphrodite array. A Hamamatsu ImagEM CCD camera (Hamamatsu) mounted on a compound microscope or a stereomicroscope recorded movies. From observations of these recordings, we scored the following mating metrics: number of times a male contacted the vulva, total duration of vulval contact, and the time a male spent scan- 
ning a hermaphrodite. The same group of males was used to obtain these datasets for each behavioral metric. A similar number of wild-type and mutant males were tested in parallel on the same day for statistical comparisons. Graphpad Prism 5 (version 4.03) software was used to perform all statistics. Mann-Whitney nonparametric test was used to compare noncategorical metrics of mutant or transgenic males versus control groups. Fisher's exact test was used when comparing categorical variables (protracted vs nonprotracted, potent vs nonpotent, etc.).

To score for mating potency, $10 \mu \mathrm{l}$ of a saturated $E$. coli culture was spotted onto a NGM agar plate, to make a $3 \mathrm{~mm}$ bacteria lawn; $\sim 20 \mathrm{~h}$ later, we placed a single male and a single adult virgin pha-1(lf) hermaphrodite onto the mating lawn and incubated them at $20^{\circ} \mathrm{C}$. A male was considered potent if we directly observed cross-progeny on the assay plate on subsequent days.

The spicule movements of wild-type or unc-7(rg396) males copulating with heat-shocked paralyzed hermaphrodites were digitally recorded with Hamamatsu ImagEM camera at a rate of $\sim 20$ frames per second. The recordings were reviewed to find an interval where the male repetitively prods the vulva with his spicules for an uninterrupted duration of $\geq 6 \mathrm{~s}$. Those frames of the recording were later analyzed using the SimplePCI software. A rectangular ROI was drawn over the male spicule region, and another over the vulva of a heat-shock hermaphrodite, to normalize the spicule movement against any slight hermaphrodite displacement. In each frame, both ROIs were used to obtain the SD of the mean pixel intensity. The mean SD of the hermaphrodite ROI was used to correct the SD for each spicule ROI frame. The oscillation amplitudes were then plotted against time. For statistical comparison, the mean pixel fluxes per second during $5 \mathrm{~s}$ were calculated for individual males from each group.

The vulva index of prodding (VIP) was calculated for mutant, transgenic, and control groups. After placing the paralyzed hermaphrodites on the small OP50 lawn, a $2 \mathrm{~cm}$ square chunk from the NGM plate was placed on a microscope slide. Prodding attempts were visualized using the $20 \times$ objective on an Olympus BX51 microscope (Olympus) and digitally recorded using the Hamamatsu ImagEM camera at $\sim 20$ frames per second for $\sim 3 \mathrm{~min}$ or until insertion was achieved. These movies were prescreened to find a consecutive interval where animals preferentially placed their tail over the vulva lips area without inserting for $60 \mathrm{~s}$. These intervals were more difficult to find for UNC-7 defective animals because these males move off the vulva more often than wild-type (see Fig. 4). Nonetheless, we still found a small group of rg396 and Pdop-2: UNC7(A59T) males where the total time in contact with the vulva was not significantly different compared with their respective controls $(5$ wild-type vs $6 \mathrm{rg} 396$ animals, average $=59$ vs $52 \mathrm{~s}$, respectively, $p=0.23$; 5 control (Pdop-2:UNC7) 7 Pdop-2:UNC7(A59T) animals, average $=58$ vs $50 \mathrm{~s}, p=0.63$; Mann-Whitney nonparametric test). Thus, we observed these "at vulva" mating intervals frame by frame to account for the time that the spicule tip contacted the hermaphrodite vulva slit or adjacent vulva slit areas. The interval where the spicule prodded at adjacent vulva slit areas was then subtracted from the interval of spicule thrusts at the slit and subsequently divided by the total time that the male tail was in contact with the vulva. A score of 1 indicates exclusive spicule thrusts at the vulva slit, and a score of -1 indicates only adjacent vulva slit spicule thrusts for the time a male attempts prodding during the $60 \mathrm{~s}$ interval.

We scored for the percentage of time an animal spent crawling backwards through observations of the general locomotion of 1-day-old unc$7(\mathrm{rg} 396)$ and control hermaphrodites. On the day of the assay, a single hermaphrodite was placed on a plate without food for $1 \mathrm{~min}$ to let the animals crawl out of the bacteria; subsequently using a pipette loaded with water, the animal was moved onto another plate without OP50. After 1 min of acclimation, we digitally recorded their locomotion at $\sim 20$ frames per second for $2 \mathrm{~min}$. These movies were then watched to score for continuous intervals where an animal reversed during a $60 \mathrm{~s}$ observation.

$\mathrm{Ca}^{2+}$ imaging and optogenetics. Experimental details for using the Mosaic illumination targeting system (Andor Technology) have been previously published (Correa et al., 2012). In brief, for optogenetic analyses of the PCS, the rgEx625 [Punc-17:ChR2::YFP, Peat-4: GCaMP6::SL2:::mDsRed, pha$1(+)$ ] and rgEx601 [Punc-17: GCaMP6::SL2:::mDsRed, Peat-4: ChR2::YFP, pha-1(+)] L4 males were incubated with all-trans-retinal for $\sim 4 \mathrm{~h}$ or until they became adults. These animals were immobilized between a microscope coverslip and an $8 \%-10 \%$ noble agar pad-containing Polybead polystyrene $0.1 \mu \mathrm{m}$ microspheres (Polysciences) (Kim et al., 2013). The males were then observed with a $40 \times$ objective on an Olympus IX81microscope fitted with the Mosaic illumination targeting system. The MetaMorph microscopy automation and imaging analysis software (Molecular Devices) allowed a $5 \times 5$ square illumination region to be specified nearby the PCA or PCB areas. To distinguish between PCA and PCB, we first observed the mounted males under DIC. To determine sufficient GCaMP6::SL2:::mDsRed transgenic expression, we briefly observed these cells under filtered green light. To increase $\mathrm{mDsRed}$ signal detection, we fitted an additional epifluorescent light source to our microscope that provided whole-field green-light illumination. MetaMorph software was used to control the Mosaic-targeted illumination system mirrors to reflect blue and green excitation light to the GCaMP6/mDsRed-expressing neuron for $\sim 15 \mathrm{~s}$, followed by directing the illumination to both ChR2-expressing and G-CaMP6-expressing neurons for $\sim 30 \mathrm{~s}$. The GCaMP6 and mDsRed fluorescence signals were recorded simultaneously using Dual view simultaneous image splitter (Photometrics) and an Andor Technology iXon EM CCD camera or an Andor Technology Zyla 5.5sCMOS, at $\sim 3$ frames per second. After the recordings, the immobilized males were unmounted from the microscope slide and immediately placed on an NGM plate. If males did not crawl on the plate, the data were discarded.

The fluorescence data were analyzed using the SimplePCI software and Microsoft Excel. Any artifacts, such as focusing, slight spicule movement, mercury arc lamp flicking, and/or photobleaching, were corrected in every frame through analyzing the red signal as previously reported (Correa et al., 2012). In brief, the red signal for each frame was calculated by subtracting mean pixel intensity (MPI) of the red channel background from the MPI red channel cellular signal. This subtracted MPI value was plotted over time. A fitted average or one-phase decay curve line was fitted against the red channel traces using GraphPad Prism. These average red values were used to normalize any artifact fluorescent changes and maximize the detection for green fluorescent changes that represent calcium transients. The final $\% \Delta \mathrm{F} / \mathrm{F} 0$ were calculated and plotted against time for each frame. The arbitrary F0 value was determined as the fluorescence value in the first frame of the recordings.

Cellular ablations. To address the HOA sensilla contribution to either the DA + ARE drug sensitivity or to the PCA to PCB target illumination response, we laser ablated HOA in paralyzed wild-type males. We used a Spectra-Physics VSL-337ND-S nitrogen laser attached to an Olympus BX51 microscope via the MicroPoint laser focusing system to ablate the P10pppa cell (HOA) in early-to-mid L4 males. Both the laser-ablated and mock-ablated males were immobilized between a microscope coverslip and $4 \%$ noble agar pad containing $2 \mathrm{~mm}$ of sodium azide. Males that were used in the optogenetic experiments were placed on NGM plates for 1-2 $h$ and then transferred to an all-trans-retinol plate for 2 more hours. The ablated males used for drug tests were placed on NGM plates for $18-24 \mathrm{~h}$.

\section{Results \\ D2-like signaling reduces calcium transients in PCB during PCA stimulation}

Spicule insertion into a hermaphrodite is a task that requires a balance between sustaining and exiting repetitive intromission attempts. Upon sensing the vulva, each PCS neuron (PCA, PCB, and PCC) promotes spicule thrusts by inducing posterior sexmuscle contractions. Reciprocal synapses between the PCS neurons suggest that stimulation of one cell could further trigger activity in the others to amplify the circuitry's response to vulva cues (Fig. 1). However, after several minutes of unsuccessful penetration, this behavioral loop might become futile and further amplification should be dampened. Experimental observations suggest that this potential dampening could coincide with increased DA ray neuronal activity, which is then followed by the male terminating spicule insertion attempts and moving off the vulva. We had shown that, during vulva withdrawal, postsynaptic D2-like signaling dampens sex-muscle excitability to diminish 

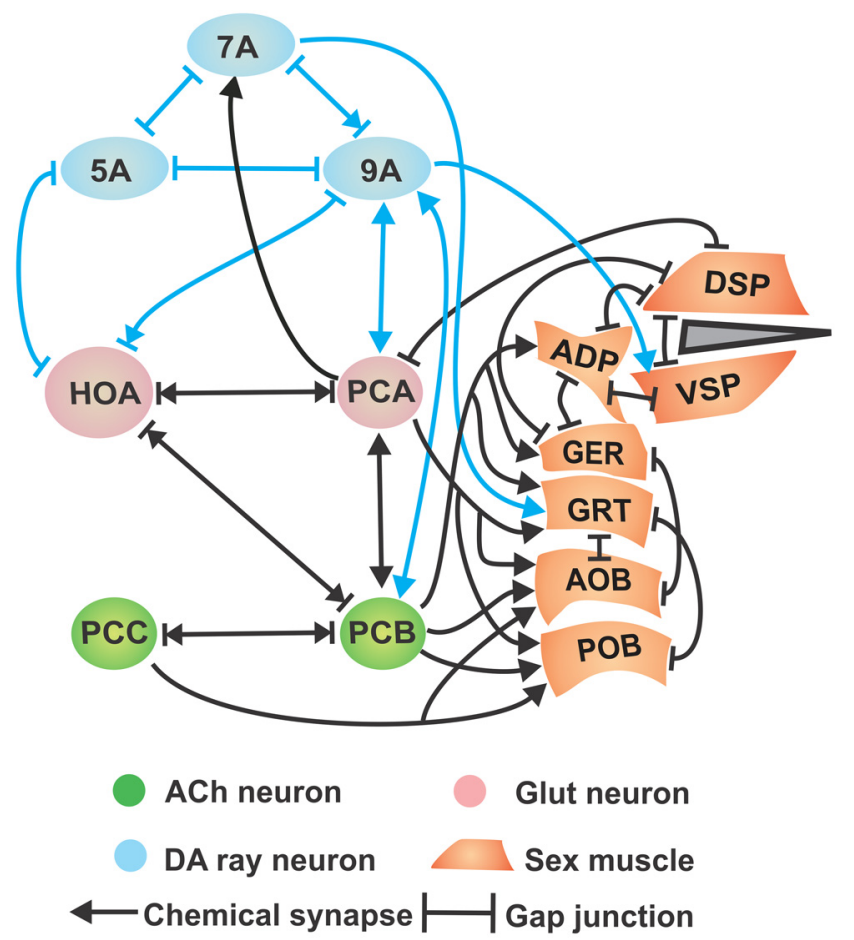

$\longleftrightarrow$ Reciprocal synapse

Figure 1. Abridged spicule intromission circuit connectome. Chemical, reciprocal, and electrical synapses among abridged neurons and/or muscles of the male mating circuit, depicted by connector arrows. Post cloacal sensilla neurons (PCA, PCB, and PCC), hook sensillum (HOA), and dopaminergic ray neurons (R5A, R7A, and R9A). Sex muscles: gubernaculum erector (GER), gubernaculum retractor (GRT), anterior oblique (AOB), posterior oblique (POB), dorsal spicule protractor (DSP), ventral spicule protractor (VSP), and anal depressor (ADP). Arrows indicate bilateral connections. Gray represents the copulatory spicule.

unproductive spicule thrusts (Correa et al., 2012); however, how DA also down-modulates reciprocal signaling between the PCS neurons was not examined in detail.

Three possible mechanisms could regulate the PCS excitability that coordinates spicule thrusting with vulva cues: (1) a selfamplifying interaction established through the reciprocal excitation between the PCS neurons; (2) a directed or reciprocal downregulating interaction between the cells; or (3) a combination of the above two hypotheses (Jarrell et al., 2012). In other words, the system might be recurrent, where the activity of one sensilla stimulates the other, but requiring extrinsic modulatory regulation; or either sensilla may be capable of directly downmodulating the other's activity, indicating that communication between them is sufficient for delimiting intromission attempts.

To distinguish between these possibilities, we assessed how single-cell stimulation affects its reciprocal partner's activity. In one cell (either PCA or PCB), we stimulated the light-activated cation channel, channelrhodopsin-2 (ChR2), with blue light (Nagel et al., 2005). At the same time, we measured cellular activity in the other neuron by imaging fluorescent changes of the calcium sensor GCaMP6 (Chen et al., 2013). To temporally and spatially confine the epifluorescent light to distinct neurons in the field of view, the male was immobilized on a microscope fitted with the Mosaic Target Illumination and Imaging System (Andor Technology). This illumination system targets light to one or many user-defined regions of interest (Correa et al., 2012). We expressed GCaMP6 or ChR2 in PCA using the glutamatergic reuptake transporter Peat-4 and in PCB using the synaptic ve- sicle acetylcholine transporter Punc-17. If the circuit is selfamplifying, we expected to see an increase in calcium transients in one neuron, upon stimulation of the other. However, if the stimulatory signal is unidirectional or the cells negatively regulate each other, we did not expect to see cross stimulation. We found that, when we photo-stimulated PCB, PCA showed increased calcium transients (Fig. 2A). However, when PCA was stimulated, PCB did not change its activity (Fig. 2B). These results suggest that $\mathrm{PCB}$ can stimulate $\mathrm{PCA}$, but $\mathrm{PCB}$ 's response to PCA might be nonexistent, inhibitory, or negatively modulated by external factors. Because PCB expresses DOP-2 (Correa et al., 2012), we asked whether DA externally down-modulates PCB activity, thus preventing the neuron from responding to PCA. Therefore, to relieve the potential DA regulation from $\mathrm{PCB}$, we conducted the experiment with DOP-2-deficient males. We found that, for $d o p-2(l f)$ males, PCA stimulation resulted in increased PCB calcium transients (Fig. 2C). To test whether the increased PCB excitability in dop-2 males could further potentiate the ChR2 artificial depolarization of this cell, we activated PCB in the dop-2(lf) background and measured PCA transients. We found that PCA transients in $d o p$-2(lf) and wild-type animals are similar (Fig. $2 A, D$ ), suggesting that cellular ChR2 activation cannot be bypassed by neuromodulatory perturbations. Together, these results suggest that PCA and PCB reciprocally stimulate each other; however, under certain conditions (such as in our immobilized males), a D2-like pathway dampens communication in the PCA to PCB direction.

\section{The gap-junction UNC-7 is an effector of D2-like signaling}

We next asked how the directional modulation of PCA-PCB communication is regulated by DA and DOP-2. To further identify molecular mechanisms involved in this process, we took a forward genetic approach combined with pharmacological analysis to identify molecules that are downstream of, or parallel to, D2-like signaling. The nonspecific ACh-agonist ARE can induce spicule protraction by stimulating nicotinic and muscarinic $\mathrm{ACh}$ receptors in the sex-muscles and spicule circuit neurons (Liu et al., 2011; Correa et al., 2012). However, exogenous DA mixed with ARE inhibits the spicule protraction response (Correa et al., 2012). Loss-of-function mutations in $d o p-2$ result in males that protract their spicules in response to DA and ARE (55\% vs $11 \%$, dop-2 vs controls, $p=0.0003$, Fig. $3 A$ ), suggesting that these mutant males are insensitive to DA down-modulation. Thus, mutations in other molecules that impair DA signaling should result in similar drug resistance.

To screen for potential molecular candidates that transduce DA signaling, we isolated EMS-induced mutant lines and scored for increased number of males with protracted spicules under DA + ARE exposure. We identified the rg396 mutant strain, where a significantly higher number of males protracted their spicules in the drug bath (67\% vs $11 \%$, rg396 vs controls, $p<0.0001$; Fig. $3 A$ ). Additionally, these mutant males have a higher percentage of constitutively protracted spicules ( $43 \%$ vs $5 \%$ mutant vs control, $p=0.0004$; Fig. $3 B$ ). Through whole genome sequencing and classical genetic mapping, we identified the rg396 allele as a missense mutation (A59T) located in the first exon of the gapjunction gene, unc-7 (Fig. 3C). The unc-7 gene encodes two types of proteins: UNC-7 long (L) and UNC-7 short (S) isoforms. The rg396 mutation affects the UNC-7(L) isoform, but not UNC$7(\mathrm{~S})$. Both isoforms can form a heteromeric gap-junction with the UNC-9 subunit to electrically couple interneurons, motor neurons, and muscles of several C. elegans sensory-motor circuits. In the locomotor circuit, these gap-junctions mediate sinu- 
A

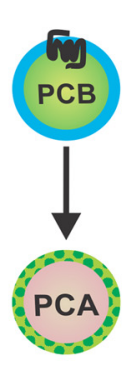

B

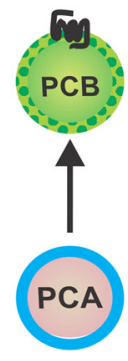

C

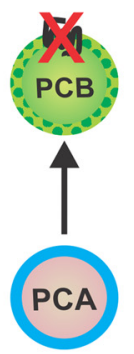

D

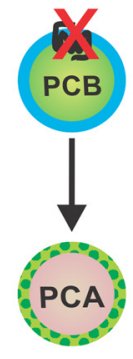

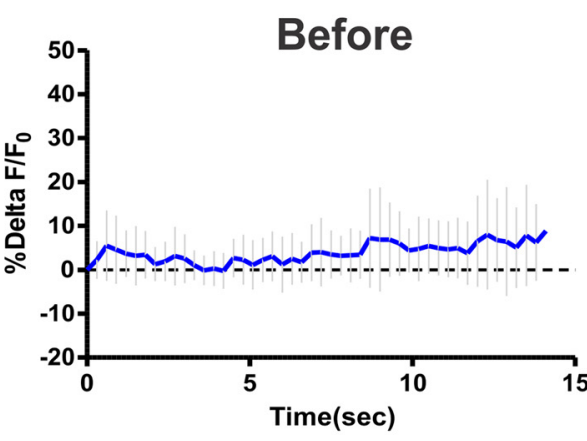
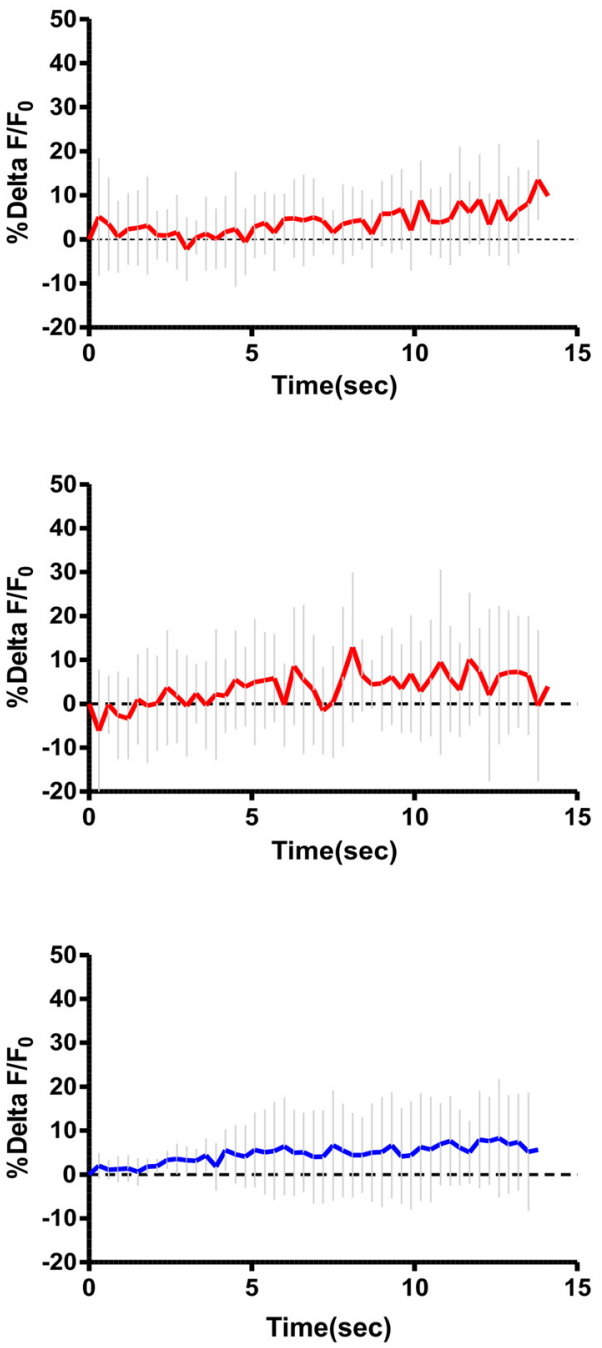
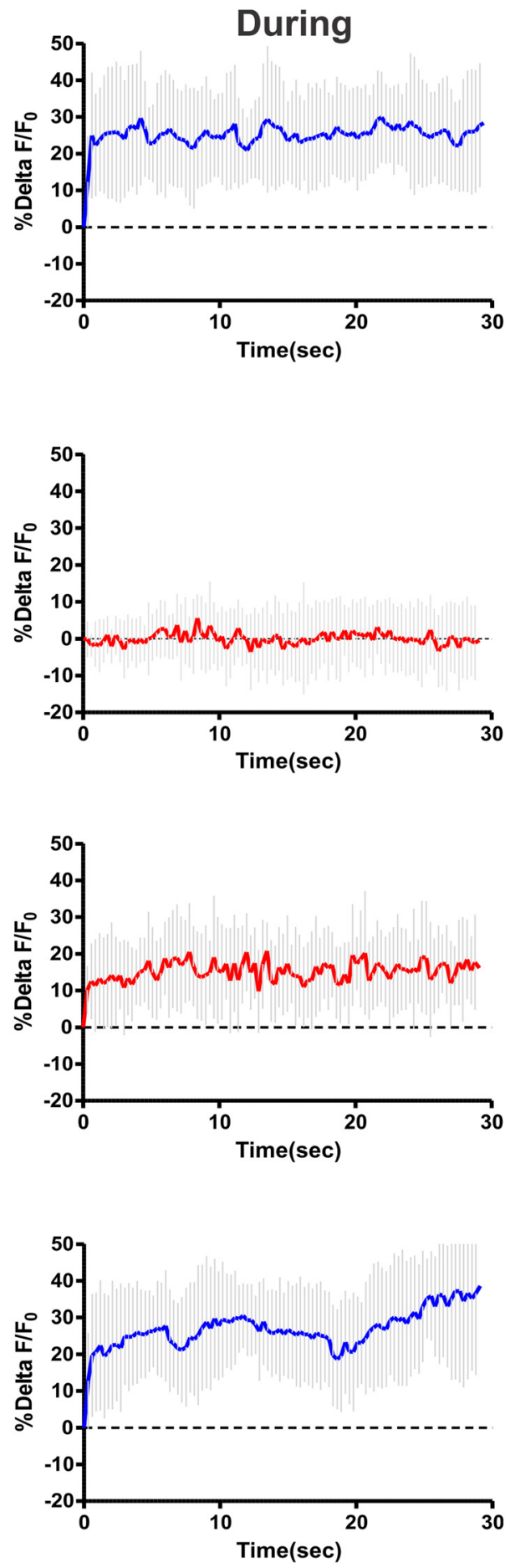

\section{ACh neuron Glut neuron}

Figure 2. D2-like signaling regulates recurrency in the $P(A$ to $P C B$ direction. $A-D, P C A$ and $P C B$ targeted illumination diagram. The directionality tested is described by the arrowhead. Green dotted and the blue outlines indicate selective GCaMP6 and ChR2 expression (left panels). The average $\% \Delta \mathrm{F} / \mathrm{F}$ calcium transients determined from all tested males before (middle panel) and during photo-stimulation of the respective cell (right panel). Light gray lines indicate the SD. $A, P C B$ to PCA stimulation in wild-type $(n=8) . B, P C A$ to PCB stimulation in wild-type $(n=10)$. $C, P C A$ to $P C B$ stimulation in dop-2 (If) males $(n=10)$. D, PCA to PCB stimulation in dop-2 (If $)$ males $(n=8)$.

soidal movement and preferential forward locomotion (Starich et al., 1993, 2009; Kawano et al., 2011).

Loss-of-function mutations in gap-junctions, such as UNC-7 and UNC-9, can affect chemical neurotransmission properties in C. elegans and vertebrate models (Yeh et al., 2009; Wang and
Belousov, 2011). Thus, perturbing UNC-7(L) function with the A59T mutation could directly alter responses related to ACh, instead of DA signaling. To address this issue, we calculated the effective concentration at which $50 \%$ of males protract their spicules $\left(\mathrm{EC}_{50}\right)$ in ARE; however, there was not a significant differ- 

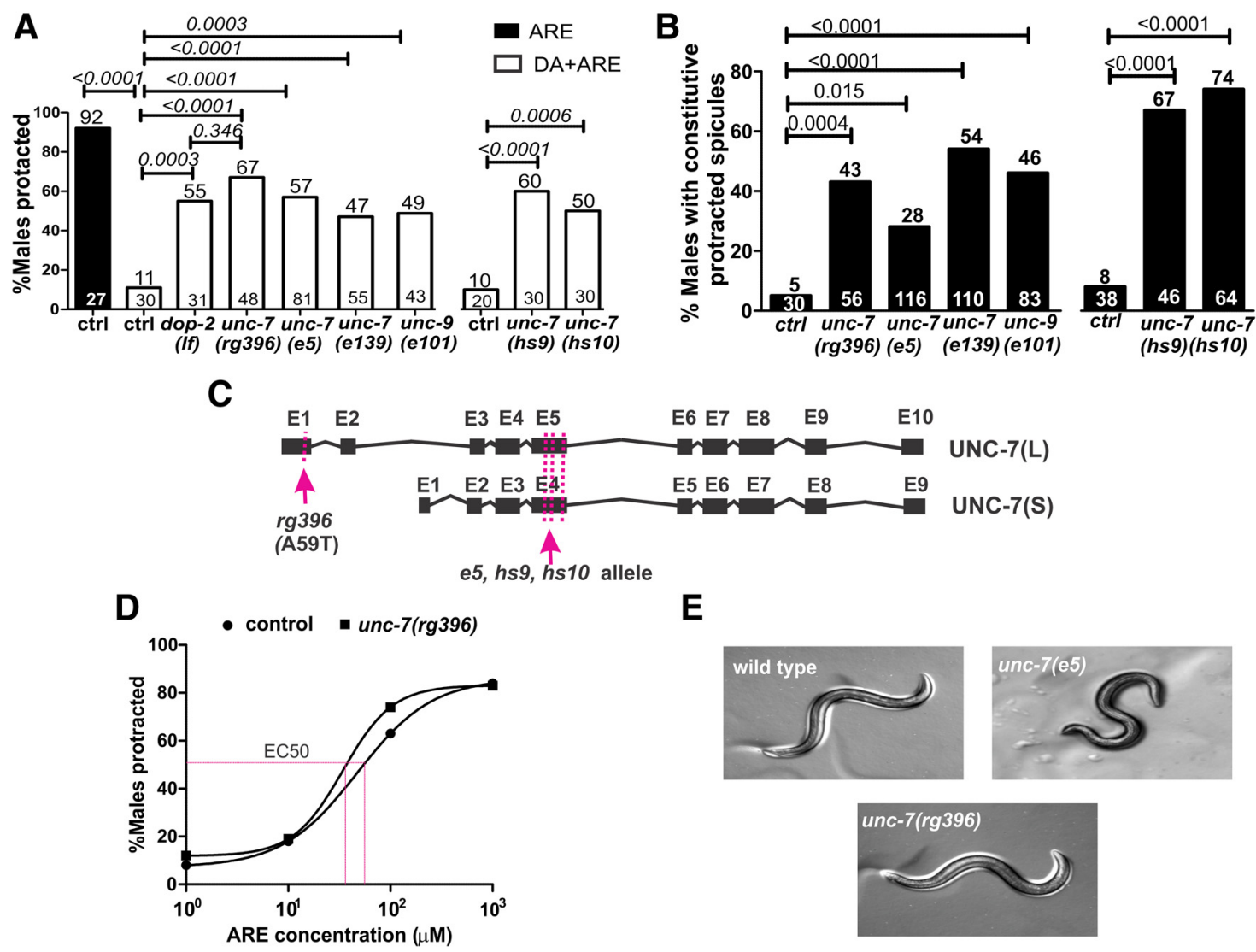

Figure 3. A point mutation in UNC-7 perturbs DA inhibition of ARE-induced protraction. $\boldsymbol{A}$, Percentage of male protraction in $20 \mathrm{~mm}$ DA $+1 \mathrm{~mm}$ ARE for control and mutant males. $\boldsymbol{B}$, Percentage of males with constitutive protracted spicules for control and unc -7 and unc- 9 mutants. Top and bottom numbers indicate the percentage and number of animals tested, respectively. The exact $p$ values were calculated using Fisher's exact test and are indicated above the black connector lines. $C$, Diagram of the UNC-7 isoforms. Black boxes and lines represent predicted exons and introns in unc-7. Magenta arrows and dotted lines indicate the location of unc-7 mutations. D, Dose-response curve of ARE-induced protraction for control and unc-7( $\mathrm{rg} 396)$ males. Each data point represents 20 or more males tested for the indicated concentration. The calculated $\mathrm{EC}_{50}$ is $47.4 \mu \mathrm{m}$ and $34.2 \mu \mathrm{m}$ for wild-type and unc-7(rg396), respectively. $\boldsymbol{E}$, Images depicting representative postures of wild-type (top), unc-7(rg396) (middle), and unc-7(e5) (bottom) males.

ence in the $\mathrm{EC}_{50}$ among $u n c-7(\mathrm{rg} 396)$ and control males (47.4 $\mu \mathrm{M}$ and $34.2 \mu \mathrm{M}$, mutant vs control males; Fig. $3 D$ ). Therefore, the increased response of unc-7(rg396) males to ARE in the DA + ARE drug bath suggests that UNC-7 mediates DA modulation.

To ascertain whether cryptic linked EMS-induced mutations in the unc-7(rg396) strain contribute to the DA-resistant phenotype, we tested whether other unc-7 loss-of-function alleles also result in similar DA-signaling deficiency. These unc-7 alleles were originally isolated because of their uncoordinated-distorted "kinker"-like locomotion and resistance to volatile anesthetics (Brenner, 1974; Starich et al., 1993; Hecht et al., 1996; Barnes and Hekimi, 1997). We surveyed the $e 5, e 139, h s 9$, and $h s 10$ alleles and found that, similar to unc-7(rg396) mutants, these mutations rendered males resistant to DA-inhibition of ARE-induced protraction (Fig. 3A), suggesting that UNC-7 gap-junctions can mediate DA-signaling. Because UNC-9 is expressed in spicule circuit neurons and muscles (Liu et al., 2011) and makes heterologous gap-junctions with UNC-7 in the locomotor circuit (Starich et al., 2009; Kawano et al., 2011), we hypothesized that unc-9(lf) males might also be DA + ARE insensitive. Consistent with this idea, we found that the unc- $9(l f)$ males have higher spicule protraction in this drug bath (Fig. 3A). Additionally, all unc-7(lf), unc-9(lf), and unc-7(rg396) mutant males displayed constitutively protracted spicules, suggestive of heightened spicule circuit excitability in these backgrounds (Fig. 3B). Unlike the severe uncoordinated phenotype typical of unc-7(lf) and unc-9(lf) ani- mals, the unc-7(rg396) males do not have gross posture or locomotion defects (Fig. 3E). However, when we quantified the incidence of backward crawling in rg396 hermaphrodites, we noticed that these animals subtly reverse more often than wild-type during a 60 s observation ( $22 \%$ more often vs $7 \%$; $r g 396$ vs wildtype, $n=10$ and 11 , respectively, $p=0.006$, Mann-Whitney nonparametric test). Together, these results suggest that the rg396 allele perturbs the mating circuit's excitability more than other circuits in the worm.

Next, we attempted to mitigate the defective drug responses in the unc-7(rg396) and a loss-of-function unc-7 allele by overexpressing wild-type UNC-7. We chose to examine the unc-7(e5) allele because the premature stop codon of this background results in an unc-7-null (Starich et al., 1993, 2009). Surprisingly, we found that unc-7(rg396) males carrying the unc- $7(+)$ transgene had similar DA + ARE sensitivity as the control strain ( $65 \%$ vs $70 \%$, respectively; Table 2); however, transgenic expression restored the unc-7(e5) lesion drug response to wild-type levels ( $57 \%$ vs $18 \%, p<0.005$; Table 2 ). These observations suggested that the rg396 allele might be a dominant negative mutation. Because the unc-7 locus is on chromosome $\mathrm{X}$ and males only have one copy of this chromosome, we could not genetically test for rg396 dominance in males. Therefore, we tested whether a single copy of this allele was sufficient to induce a locomotor deficit in the hermaphrodite, similar to the rg396 homozygous animals. We found that heterozygous hermaphrodites had a higher inci- 
Table 2. D2-like signaling inhibits ARE-induced protraction via UNC-7 in neuronal tissues

\begin{tabular}{|c|c|c|c|}
\hline Genotype & $\begin{array}{l}\text { DA + ARE } \\
(\%) \text { SpOUT }(n)\end{array}$ & $p$ & Compared with \\
\hline Control & $11(30)$ & & \\
\hline unc-7 (rg396) & $65(48)$ & 1 & dop-2; rg396 \\
\hline dop-2 (If) & $55(31)$ & 0.632 & dop-2; rg396 \\
\hline unc-7 (rg396); dop-2 & $63(43)$ & $1,0.632$ & dop-20R rg396 \\
\hline unc-7 (rg396); pha-1 & $65(85)$ & 1 & unc-7(rg396) \\
\hline $\begin{array}{l}\text { unc-7 (rg396); pha-1; rgEx644 (UNC-7: } \\
\text { YFP)TL1 }\end{array}$ & $70(44)$ & 0.657 & unc-7(rg396); pha-1 \\
\hline unc-7(e5);pha-1 & $57(81)$ & 0.459 & unc-7(rg396) \\
\hline $\begin{array}{l}\text { unc-7(e5);pha-1;rgEx653 (UNC-7:YFP; } \\
\text { SL2::GFP) }\end{array}$ & $18(61)$ & 0.007 & unc-7;pha-1 \\
\hline pha-1;rgEx653 (UNC-7:YFP; SL2::GFP) & $22(91)$ & & \\
\hline pha-1; rgEx668 (UNC-7:YFP) ${ }^{a}$ & $40(59)$ & 0.017 & pha-1,rgEx653 \\
\hline pha-1; rgEx672 (Plev11:UNC-7:YFP) & $17(69)$ & 0.552 & pha-1;rgEx653 \\
\hline pha-1; rgEx673 (Paex-3:UNC-7:YFP) ${ }^{b}$ & $44(81)$ & 0.002 & pha-1;rgEx653 \\
\hline $\begin{array}{l}\text { pha-1; rgEx671 (Punc-103E:UNC-7: } \\
\text { YFP) }\end{array}$ & $28(68)$ & 0.457 & pha-1;rgEx653 \\
\hline pha-1; rgEx674 (Pdop-2:UNC-7:YFP) ${ }^{b}$ & $44(62)$ & 0.0071 & pha-1;rgEx653 \\
\hline
\end{tabular}

${ }^{a}$ UNC-7 genomic with A59T mutation.

${ }^{b}$ UNC-7 (A59T) minigene. acts as a dominant negative in the mating circuit, we expressed the UNC-7(L)(A59T) version in wild-type and found that the transgenic mutant protein expressed from its promoter induced the rg396 phenotype and rendered males insensitive to DA inhibition (Table 2). Together, these results suggest that UNC-7 couples an electrical signal in the spicule circuit and that this is potentially regulated by an upstream D2-like pathway.

In vertebrate sensory circuits, D1- and D2-like signaling modulates gap-junction activity (Pereda et al., 1992; Kothmann et al., 2009; Kirkby and Feller, 2013); however, there have been no reported genetic studies that elaborate on these interactions. Because dop-2(lf) and unc-7(rg396) males have similar drug sensitivities (Fig. 3A; Table 2), these two molecules might regulate common aspects of mating circuit excitability and thus are in the same pathway. To genetically test this hypothesis, we generated a dop-2(lf); unc-7(rg396) double mutant and tested whether it and unc-7, and dop-2 single mutants have similar drug responses. We found that there was no change in the response to DA + ARE between the double and single mutant males (Table 2), consistent with DOP-2 and UNC-7 affecting the same pathway. Furthermore, these observations suggest that DA-mediated gap-junction regulation is conserved between vertebrate and invertebrate sensory-motor circuits.
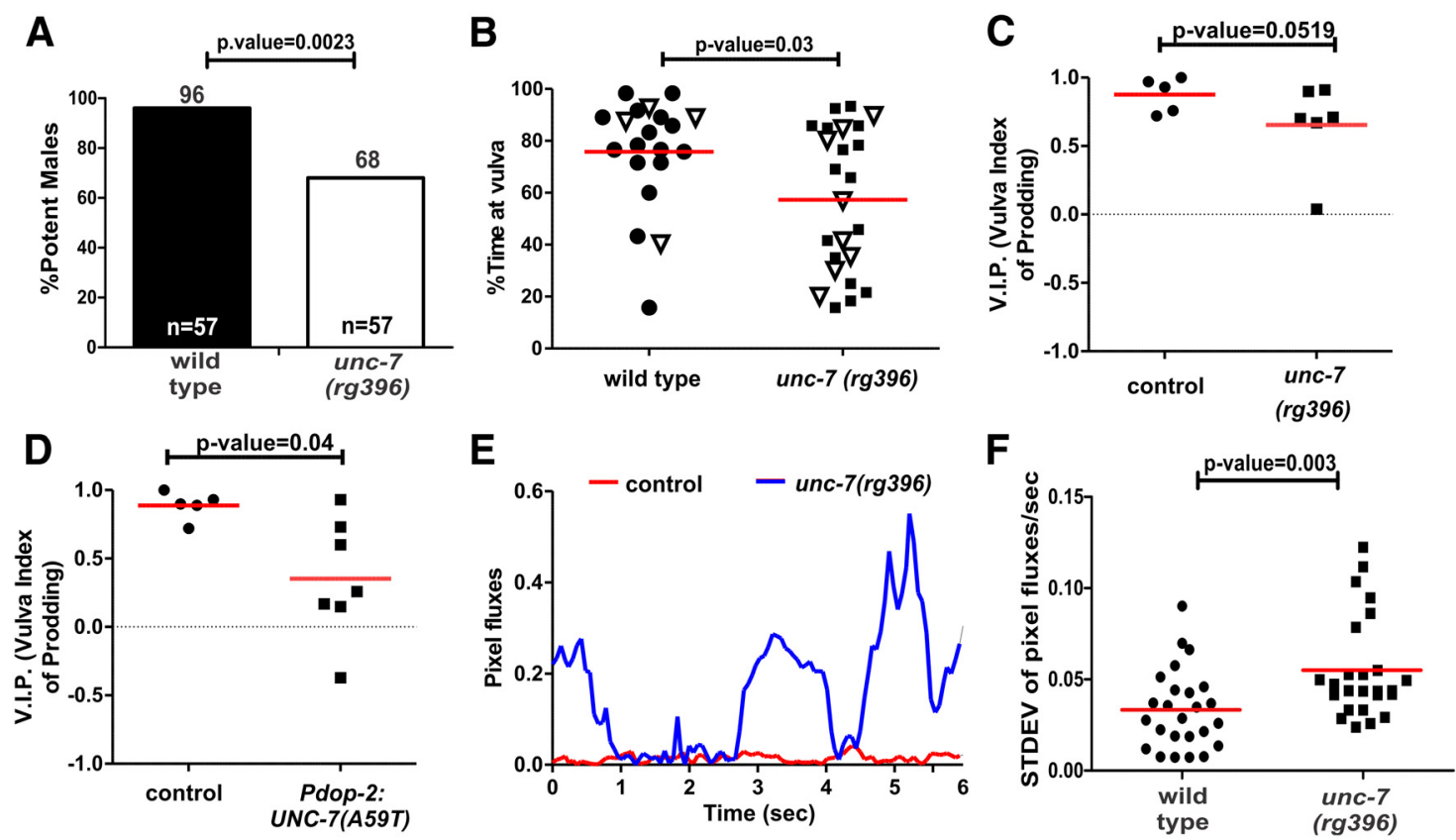

Figure 4. UNC-7 diminishes the magnitude of spicule insertion attempts at the vulva and restricts intromission attempts at nonvulva regions. Mating observations between wild-type and unc-7(rg396) males. A, Mating potency for wild-type and unc-7(rg396) with moving hermaphrodites. Numbers on top and bottom of the bars indicate percentages and number of males tested. The $p$ value was calculated using Fisher's exact $t$ test. $\boldsymbol{B}$, The percentage of time that males spent in contact with the vulva out of $120 \mathrm{~s}$ of total contact. Each symbol represents an individual's male performance. Open symbols indicate males that fully protracted their spicules out of the tail. $C, D$, VIP during a 60 s observation window of mating with nonmoving hermaphrodites. $C$, Comparison of wild-type and unc-7( $\mathrm{rg} 396)$ mutants. D, Comparison of control and UNC-7(L) (A59T) overexpression from the dop-2 promoter. $E$, A representative trace of pixel fluxes during spicule insertion attempts of a wild-type (red trace) and mutant male (blue trace) for $6 \mathrm{~s}$. $F$, Average SD of pixel fluxes per second for 5 males per group. For each male, their prodding duration was broken down to five periods. Each point represents the SD of pixel fluxes in a $1 \mathrm{~s}$ period $(\boldsymbol{B}-\boldsymbol{F})$. Red line indicates the average value for each group. The $p$ value was calculated using the Mann-Whitney nonparametric test. The exact $p$ values are indicated above the black connector lines.

dence of crawling backwards, like the rg396 homozygote ( $22 \%$ vs $15 \%$; $r g 396$ vs $r g 396 /+, n=11$ and 10 , respectively, $p=0.192$, Mann-Whitney nonparametric test). Because e5/+ animals have no locomotion deficits ( $6 \%$ vs $7 \% ; e 5 /+$ vs wild-type, $n=10$ and 11 , respectively, $p=0.522$, Mann-Whitney nonparametric test), we ruled out the possibility of haploid insufficiency in the rg396/+ background. Moreover, to confirm that the rg396 allele
UNC-7 regulates rhythmic spicule thrusts at the vulva slit and diminishes spicule intromission attempts at nonvulva areas In contrast to the pleotropic defects caused by the unc-7-null allele, the restricted unc-7(rg396) phenotype allowed us to address how the mutant allele disrupts mating behavior. When mated to moving hermaphrodites, unc-7(rg396) males had a lower ability to sire cross progeny than wild-type ( $96 \%$ vs $68 \%$, 

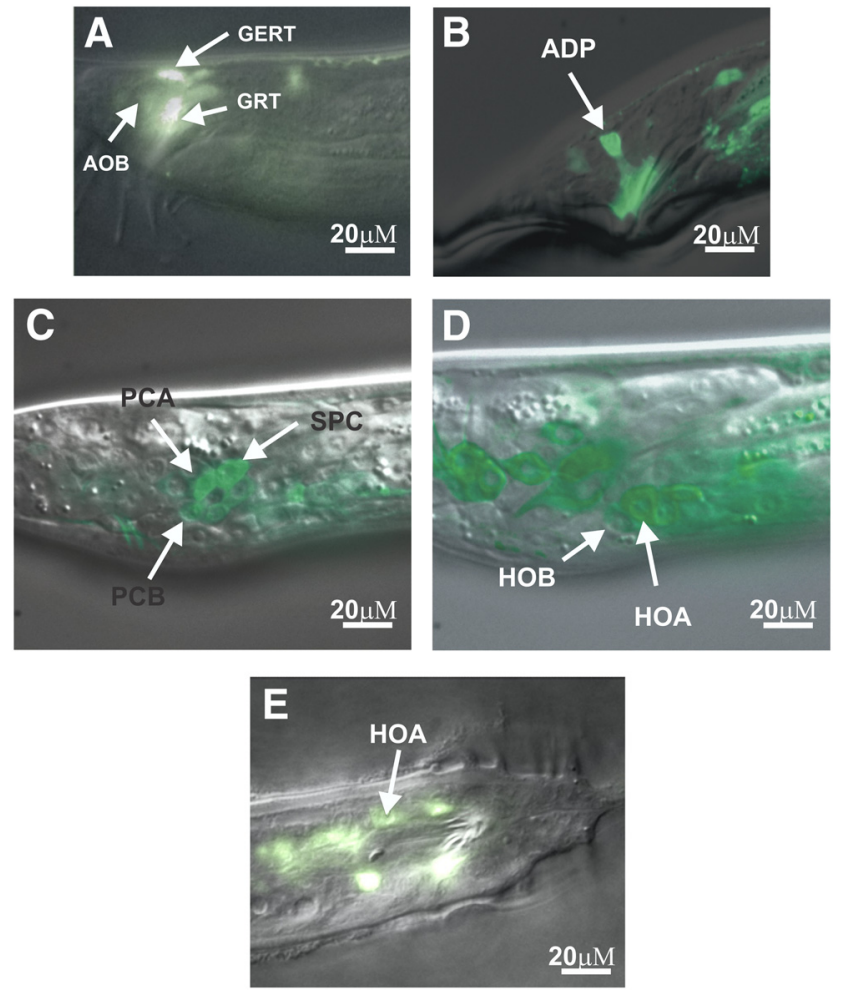

Figure 5. UNC-7 and DOP-2 expression in male-specific sensory motor neurons and copulation muscles. $\boldsymbol{A}-\boldsymbol{E}$, Merged DIC images with YFP and/or GFP fluorescence images. $\boldsymbol{A}, \boldsymbol{B}$, Adult sex-muscle Punc-7:YFP expression. Gubernacular erector (GER), gubernacular retractor (GRT), anterior oblique (AOB), and anal depressor (ADP). C, D, Punc-7:unc-7::YFP expression. C, Expression in the post cloacal sensilla neurons (PCA and PCB) and SPC sensory motor neuron. $D$, HOA and HOB expression in the $L 4$ male tail. $E$, Pdop-2:GFP expression in HOA neuron at the adult male tail.

$p=0.0023$; Fig. 4A). To determine how unc-7(rg396) might disrupt mating, we digitally recorded 1-day-old virgin unc-7(rg396) and wild-type males copulating with a nonmoving hermaphrodite for 120 s or until spicule insertion. From these recordings, we measured the number and duration of male tail contacts with the vulva. Similar to the reported DA-deficient male phenotypes (Correa et al., 2012), we found that unc-7(rg396) males spent on average less time in contact with the vulva than in contact with other parts of the hermaphrodite ( $75 \%$ vs $56 \%, p=0.03$; Fig. $4 B$ ). From the recordings, we suspected that the mutants hesitate at nonvulva cuticle regions engaging in nonproductive spicule insertion attempts. To quantify nonvulva spicule prodding events, we calculated a VIP during a $60 \mathrm{~s}$ period of male tail contact with the vulva area. A VIP score of 1 indicates exclusive spicule thrusts at the vulva slit, and a score of -1 indicates prodding at areas adjacent to the vulval slit. We found that unc-7(rg396) males had slightly lower VIP scores than wild-type ( 0.66 vs 0.88 , respectively, $p=0.052$, Mann-Whitney nonparametric test; Fig. $4 C$ ); however, this difference was not significant. Nonetheless, these differences were exacerbated in UNC-7(A59T) transgenic males, where expression of the mutant molecule was restrictively driven from the $d o p-2$ promoter $(0.35$ vs 0.89 , respectively, $p=0.04$, Mann-Whitney nonparametric test; Fig. 4D). These results indicate that normally wild-type UNC-7 acts to diminish PCS activity when the males are not at the vulval slit.

We additionally reported that, during prodding behavior, D2like signaling is necessary to maintain rhythmic spicule thrusts over the vulva slit. Males lacking the dop-2 and dop-3 D2-like receptors displayed erratic spicule prodding behavior when they attempted to breach the vulva; premature sustained thrusts were interspersed with shallow rapid spicule prods. To assess whether unc-7(rg396) also disrupted spicule rhythms, we recorded spicule motion during intromission behavior and measured pixel fluxes of the recordings at the area of contact between the spicule tips and the vulva slit (Correa et al., 2012). The pixel fluxes are a proxy for the motor output magnitude; greater values indicate more exaggerated motion. As expected, we found that the rhythmic spicule movements of unc-7(rg396) males were more vigorous (Fig. 4E) and more erratic than wild-type (Fig. 4F). These results suggest that the male uses UNC-7, similar to D2-like receptors, for regulating the timing, rhythm, and intensity of spicule insertion behavior at the vulva.

\section{UNC-7 functions in DOP-2-expressing cells that control spicule behavior}

We previously reported male-specific DOP-2 expression in sex muscles and in the cholinergic neuron PCB (Correa et al., 2012); however, there are no reports of UNC-7 expression in the male tail. In the hermaphrodite, UNC-7(L) and (S) isoforms (Fig. 3B) are broadly expressed in all premotor and motor neurons (Altun et al., 2009; Starich et al., 2009; Yeh et al., 2009; Kawano et al., 2011). Additionally, posterior body wall muscles showed UNC-7 fluorescent immunolabeling (Yeh et al., 2009; Kawano et al., 2011). To determine which tissues UNC-7 and DOP-2 overlap, we assessed the reported $\mathrm{UNC}-7(\mathrm{~L})$ and $(\mathrm{S})$ expression pattern in the male. The UNC-7(L) and (S) reporter constructs include $\sim 1$ $\mathrm{kb}$ and $3.3 \mathrm{~kb}$ upstream sequences from their respective first introns fused to YFP (Altun et al., 2009). We found no and/or very dim expression in male-specific tissues with these reporters. We reasoned that the arbitrarily chosen upstream sequences of the UNC-7 isoforms represented limited promoter regions; therefore, we constructed more comprehensive reporters. When we increased the promoter region of UNC-7(L) up to $5 \mathrm{~kb}$, we found expression in sex muscles (anal depressor, gubernacular erector, gubernacular retractor, and anterior oblique; Fig. $5 A$ ). Because unc-7 has large introns (intron 2 and 5; Fig. 3C), we reasoned that there might also be enhancer elements in these regions in addition to more upstream promoter regions. Therefore, we further extended the UNC- 7 (L) promoter to $\sim 8 \mathrm{~kb}$ upstream of the first exon and included the entire unc-7 genomic region fused to YFP. In these transgenic males, we found UNC-7 expression, in addition to the mentioned sex muscles, in several mating circuit neurons (SPC, PCA, PCB, HOA, and unidentified ray neurons; Fig. $5 B-D$ ). Similar to previous studies (Starich et al., 2009; Yeh et al., 2009; Kawano et al., 2011), this reporter also expresses, in head ganglion neurons, several ventral chord neurons, and tail neurons of the hermaphrodite, in addition to few unreported pharyngeal neurons (I5, M2, I4, M3, and I1). Therefore, this broad expression pattern indicates that UNC-7 could electrically couple sex-specific muscles and/or neurons to modulate circuitry output.

To assess UNC-7's role in mediating DA signaling within the spicule protraction circuit, we asked where unc-7 functions in the DA inhibition of ARE-induced responses. Because the unc-7(rg396) lesion is dominant, we could not use the wild-type UNC-7 transgenic. Instead, we expressed the mutant UNC-7(A59T) transgene in different cells of wild-type males and measured the altered drug resistance. We found that UNC-7(L)(A59T) expression from a pan-neuronal promoter (Paex-3) (Iwasaki et al., 1997) caused an increase in spicule protraction when males were exposed to DA + ARE (44\% vs 


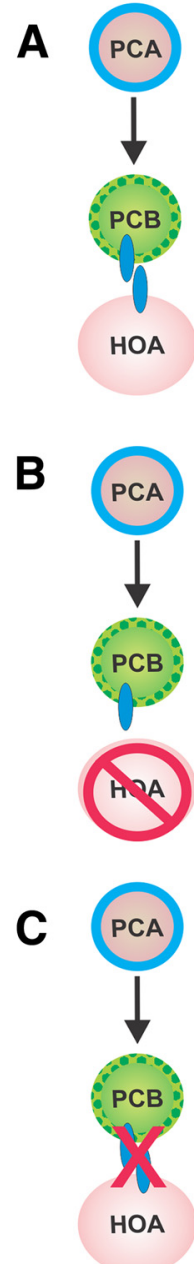

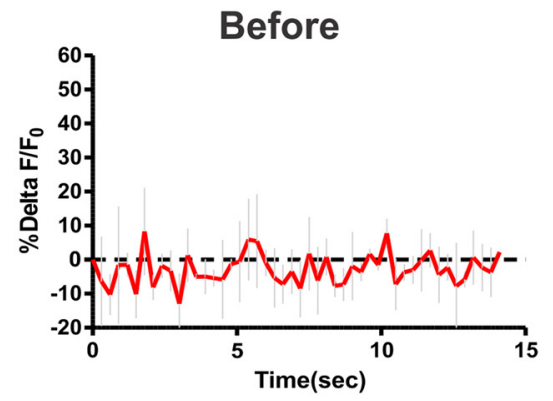
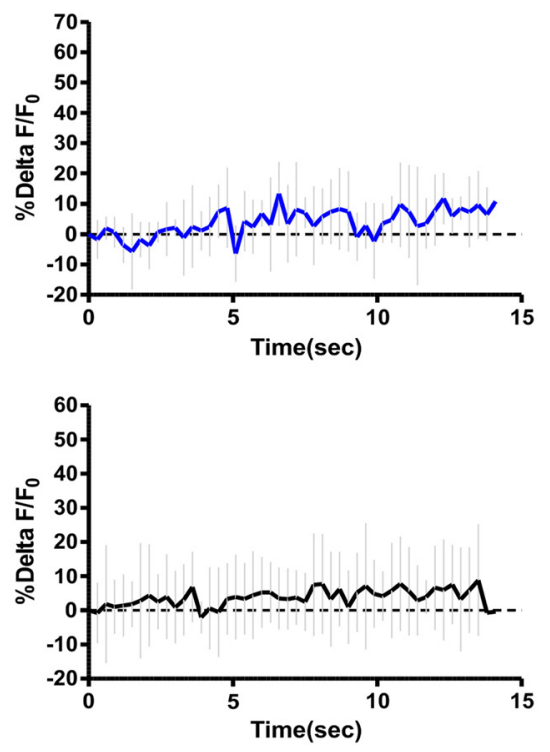

ACh neuron
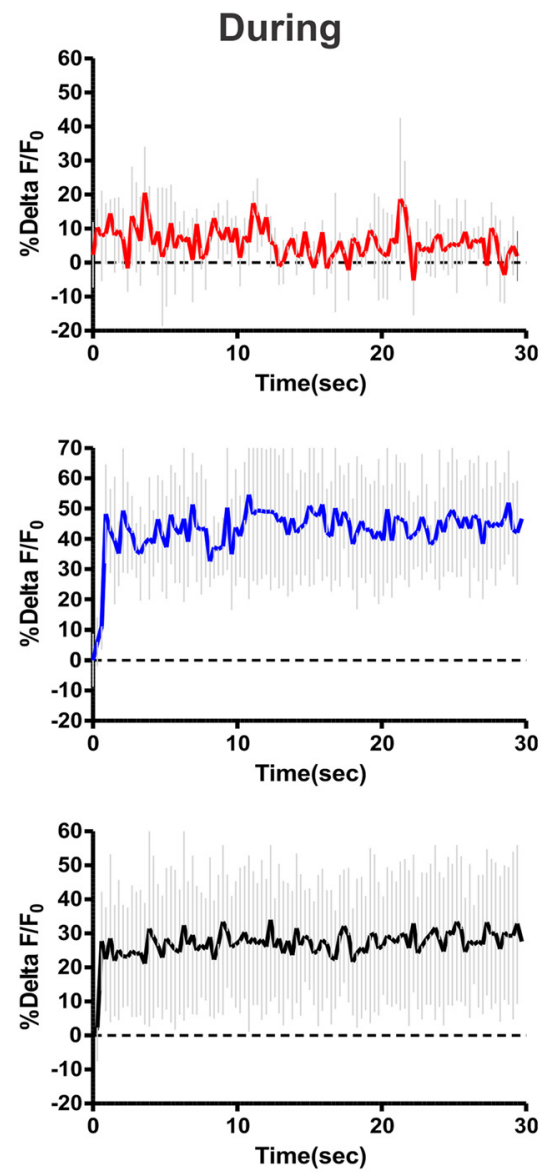

UNC-7

Figure 6. HOA-dependent regulation of PCS recurrency. A-C, PCA to PCB targeted illumination diagrams. The directionality tested is described by the arrowhead. Green dotted and the blue outlines indicate selective GCaMP6 and ChR2 expression (left panels). The average \% $\Delta \mathrm{F} / \mathrm{F}$ calcium transients determined from all tested males before (middle panels) and during PCA photostimulation (right panels). Light gray lines indicate the SD for $(\boldsymbol{A})$ mock-ablated males $(n=3),(\boldsymbol{B})$ H0A-ablated males $(n=5)$, and $(\boldsymbol{C})$ unc-7( $\mathrm{rg} 396)$ males $(n=10)$.

$22 \%$, transgenic males vs control strain, respectively, $p<$ 0.005; Table 2). In contrast, UNC-7(L)(A59T) expression from a pan-muscular promoter and sex-muscle-specific promoter (Plev-11 and Punc-103E, respectively) (Kagawa et al., 1995; Reiner et al., 2006) was unable to mimic the $r g 396$ phenotype ( $17 \%$ and $28 \%$, respectively, vs $22 \%$ for the control strain) (Table 2). These observations indicate that expressing UNC-7(L)(A59T) in neurons mimics the unc-7(rg396) lesion, thus demonstrating that UNC-7 might couple neuronal activity in the mating circuit.

Next, we asked whether UNC-7 couples the electrical activity in a neuronal subset. Because our behavioral experiments indicate that disrupting UNC-7 in DOP-2-expressing cells induces ectopic spicule insertion attempts, we asked whether UNC-7 is required directly to mediate DOP-2 inhibitory signaling in those cells. Indeed, we found that the Pdop-2:unc-7(A59T) transgenic males had increased number of protracted spicules in DA + ARE (44\% vs $22 \%$, respectively, $p<0.05$; Table 2 ). Thus, normal UNC-7(L) activity in DOP-2-expressing neurons is required for the DA + ARE wild-type response. We have already shown, through photostimulation experiments, that DOP-2 dampens PCB activity (Fig. 2), raising the question of who is PCB's gap-junction partner.

The male tail connectome indicates that HOA is a potential PCB electrical partner (Fig. 1) (Jarrell et al., 2012). Previous be- havioral observations indicate that the sensory hook neurons $\mathrm{HOA}$ and $\mathrm{HOB}$ not only detect the general vulva region but also regulate rhythmic spicule thrusts. When the hook neurons are laser-ablated, the male will not only pass over the vulva many times, but will also spontaneously prod random areas of the hermaphrodite with their spicules. This phenomenon requires the PCS neurons and has been hypothesized as an adapted response of the HOA/HOB-ablated male to use its spicules for locating the vulva orifice (Liu and Sternberg, 1995; Barr and Sternberg, 1999). Because this observation suggests that the hook neurons might negatively regulate the activity of the PCS, we reexamined DOP-2 expression in the male tail and also identified it in HOA (Fig. 5D). Therefore, we further examined whether DOP-2 might modulate PCS activity through UNC-7 gap-junctions between PCB and HOA.

\section{UNC-7 and HOA can inhibit PCA to PCB stimulation}

Similar to how DOP-2 was determined to down-modulate PCA and PCB recurrency (Fig. 1C), we used ChR2, GCaMP6, and the Mosaic targeted illumination system to dissect how coupling between HOA and PCB affects PCS excitability. We stimulated ChR2 in PCA and measured calcium transients in PCB for intact and HOA laser-ablated males before and during blue-light PCA 


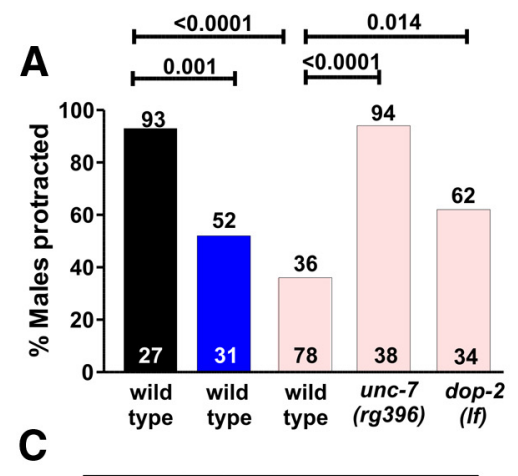

\section{B}
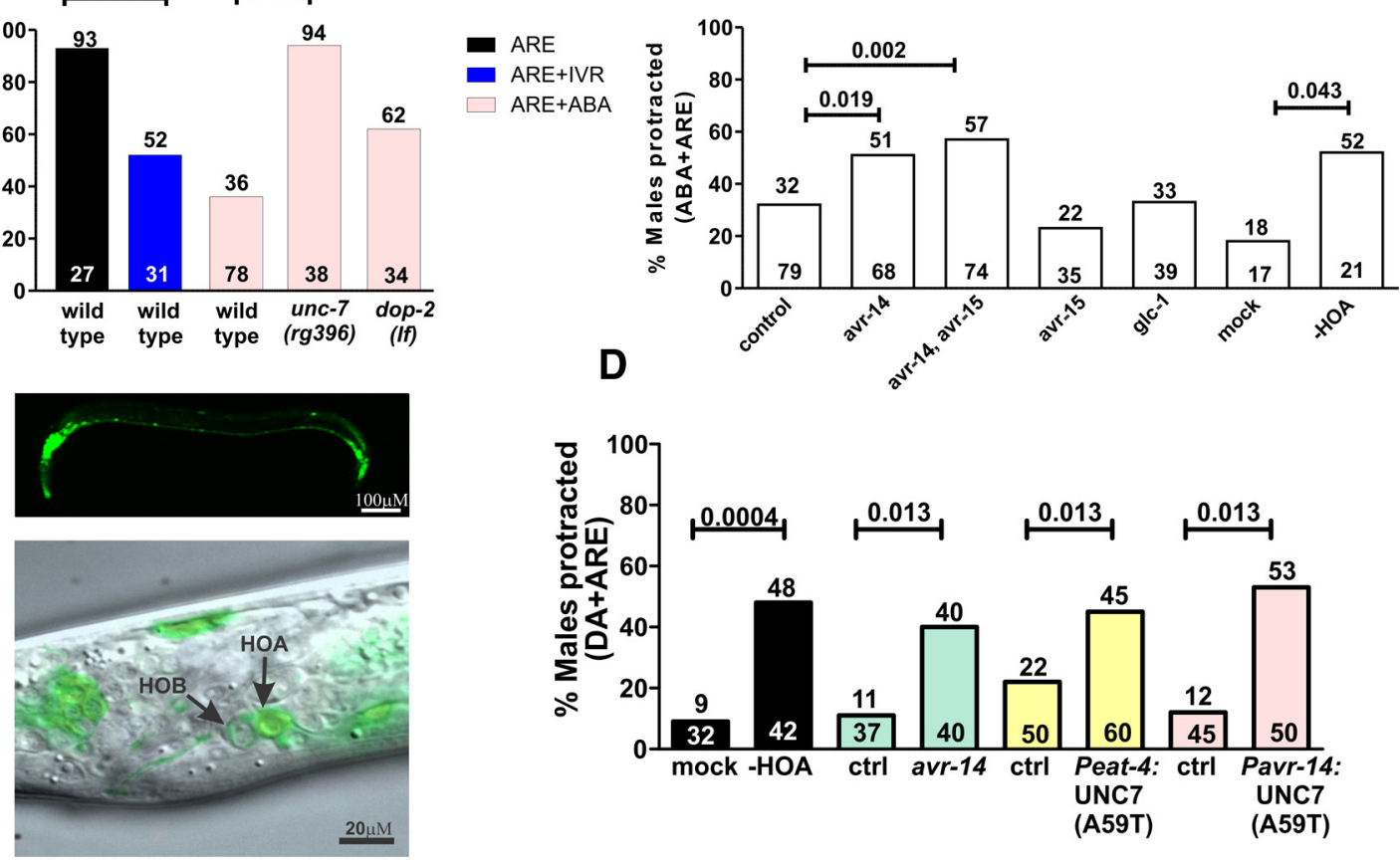

Figure 7. HOA mediates ABA inhibition of ARE-induced protraction and requires avr-14 and unc-7.A, Percentages of wild-type and mutant males that protract their spicules in the corresponding avermectin baths. $B$, Loss-of-function mutants for avr-14, avr-15, and glc-1; mock versus HOA-ablated males response to ABA + ARE. C, Expression pattern of Pavr-14:YFP in several head, ventral chord, dorsal chord, and tail neurons (top). Overlaid DIC and confocal YFP image of avr-14 expression in HOA (bottom). D, DA + ARE response for avr-14, HOA ablated males, UNC-7(A59T) tissue-specific overexpression, and respective controls. $A-D$, Top and bottom numbers indicate the percentage and number of animals tested, respectively. All $p$ values were calculated using Fisher's exact $t$ test. The exact $p$ values are indicated above the connector lines.

stimulation. As predicted, only when $\mathrm{HOA}$ was ablated, the PCB calcium transients increased (Fig. 6A,B). Next, we photostimulated ChR2 in PCA while simultaneously measuring GCaMP6-fluorescent changes in PCB before and during PCA blue light stimulation in unc-7(rg396) males. Similar to the HOA-ablated male, we found that the calcium transients in PCB also increased when UNC-7 function is compromised (Fig. 6C). These results are consistent with the idea that HOA diminishes PCB activity through UNC-7 gap-junctions during PCS stimulation.

\section{HOA requires AVR-14 and UNC-7 to relay hyperpolarizing signals onto PCS}

Our findings suggest that HOA diminishes PCB excitability through UNC-7 gap-junctions. This idea is consistent with reports suggesting that UNC-7 and UNC-9 gap-junction subunits can shuttle hyperpolarizing chloride $\left(\mathrm{Cl}^{-}\right)$currents to reduce the outputs of hermaphrodite pharyngeal, egg-laying, and locomotory circuits (Barnes and Hekimi, 1997; Dent et al., 2000; Altun et al., 2009). The UNC-7 and UNC-9 innexins mobilize $\mathrm{Cl}^{-}$to mediate the inhibitory effects of the anthelmitic drugs IVM and ABA. Both of these drugs cause general locomotory paralysis in $C$. elegans, potentially through similar signaling mechanisms (Boswell et al., 1990; Barnes and Hekimi, 1997; Ghosh et al., 2012). IVM interacts with several Cys-loop family receptors, including GABA, glutamate, and nicotinic ACh receptors (Cully et al., 1994; Hernando and Bouzat, 2014). IVM binding to GABA and/or glutamate-gated channels results in chloride influx, which is subsequently relayed via UNC-7 and UNC-9 to broader neuronal populations of pharyngeal and locomotory circuits (Boswell et al., 1990; Cully et al., 1994; Dent et al., 1997).
To address whether chloride down-modulation also occurs between HOA and the PCS network, we first tested whether IVM and/or ABA could inhibit ARE-induced protraction, similar to DA exposure. We found that mixing ABA with ARE was able to reduce spicule protraction by $60 \%$ (93\% vs $36 \%$ ARE vs ABA+ARE response, $p<0.0001$; Fig. $7 A$ ); however, IVM reduced spicule protraction more mildly (Fig. $7 A$ ). Therefore, we used the ABA+ARE drug bath to test whether UNC-7 was required for the ABA down-modulation. We found that both unc$7(r g 396)$ and dop-2(lf) males were insensitive to the inhibition (Fig. 7A), suggesting an interaction between ABA-sensitive channels, DOP-2 receptors, and UNC-7 gap-junctions in regulating ACh-induced spicule protraction.

If UNC-7 relays the $\mathrm{Cl}^{-}$current from $\mathrm{HOA}$ onto $\mathrm{PCB}$ to suppress spicule circuit excitability, then $\mathrm{Cl}^{-}$glutamatergic and/or GABA channels should function in HOA. Because PCA chemically synapse HOA and are likely glutamatergic (Jarrell et al., 2012; LeBoeuf et al., 2014), we addressed the possibility that glutamate chloride channels function in HOA to mediate the ABA response. To narrow down which inhibitory glutamate receptors function in the spicule circuit, we focused on the subset that has been shown to function in the pharynx and locomotory circuit: $a v r-14$, avr-15, and glc-1 (Dent et al., 2000; Ghosh et al., 2012). We asked which of these mutants is resistant to the ABA inhibition of ARE-induced protraction. We found that males lacking the avr-14 gene are less sensitive to the ABA inhibitory effect (Fig. 7B). Therefore, to confirm whether AVR-14 in HOA partially mediates inhibitory signaling, we examined the Pavr-14: YFP expression in the male. We found that this receptor is expressed in several head, ventral cord neurons and HOA (Fig. 7C). However, this decrease in sensitivity is much milder than the 


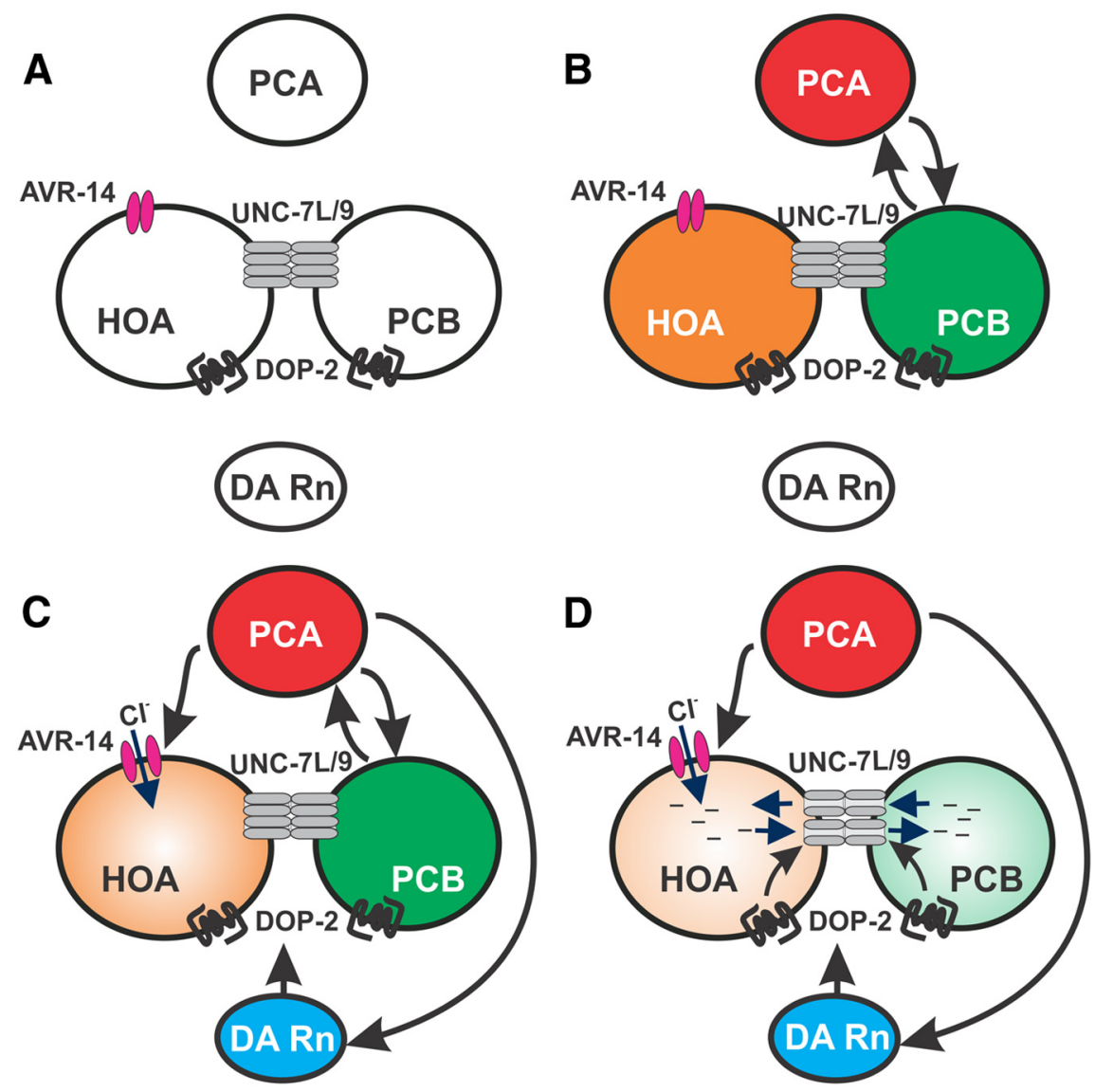

Figure 8. D2-like modulation of UNC-7 dampens PCA and PCB cross talk. Diagram depicting proposed PCS, HOA, and ray neuron activity at different stages of spicule prodding. $A$, Before reaching the vulva, cells are inactive due to the lack of vulva sensory input. $B$, When at the vulva, $H O A, P C B$, and PCA are stimulated by vulva cues, triggering PCA and PCB recurrency. $C$, Prolonged spicule prodding results in DA ray neuron activity and the activation of AVR-14 glutamate-gated chloride channels on HOA. D, DOP-2 signaling in HOA and PCB results in the opening of UNC-7L homotypic and possibly UNC-7L/UNC-9 heterotypic gap-junctions. Inhibitory signals, such as chloride $\left(\mathrm{Cl}^{-}\right)$ions, are relayed from $\mathrm{HOA}$ into $\mathrm{PCB}$, thus reducing the ability of $\mathrm{PCB}$ to be stimulated by PCA. Black arrows indicate proposed cellular interactions.

unc-7(rg396) mutant's response (Fig. 7A). The modest avr-14 resistance to $\mathrm{ABA}$ might be due to receptors with redundant function. Because C. elegans has numerous cys-loop ionotropic receptors (Jones and Sattelle, 2008), in addition to avr-14, others could also be ABA-responsive. Together, these results suggest that chloride influx occurs in HOA at least partially through AVR-14.

To confirm the hypothesis that HOA is the neuronal substrate that mediates ABA-inhibition in the spicule circuit, we laserablated HOA in wild-type males and tested for their ABA+ARE response. We found that the operated males are less-sensitive to ABA-dependent inhibition compared with mock treatments (Fig. $7 B$ ). However, again, the HOA ablation was not able to account for the high level of ABA insensitivity seen in unc7(rg396) mutant males, indicating that UNC-7 gap-junctions must regulate spicule circuit excitability in an additional subset of cells. Moreover, the DA + ARE resistance of unc-7(rg396) mutants suggests that some of the cells where UNC-7 regulates mating circuit excitability, including HOA, are under D2-like regulation. Therefore, we reasoned that males lacking HOA and loss-of-function avr-14 should also be resistant to DA downmodulation of ARE. We found that, in both instances, a higher percentage of males protract their spicules in the DA + ARE bath (Fig. 7D). Additionally, when we expressed UNC-7(A59T) in
HOA, from two different promoters (Peat-4 and Pavr-14), males were also insensitive to DA inhibition of AREinduced protraction (Fig. 7D). Together, these results are consistent with a model where activated DOP-2 results in open UNC-7 gap-junctions to allow $\mathrm{Cl}^{-}$influxes from HOA via AVR-14 to move into and reduce PCB activity (Fig. 8D).

\section{Discussion}

The persistent activity in reciprocally connected neurons of animals has been shown to regulate complex contextdependent behaviors. Many of these studies have focused on the recurrent neural interactions that occur in the mammalian cortex. Extensive studies have shown that these cortical networks use DA-activated receptors to regulate their cellular firing patterns. (Shepherd et al., 2005; Noudoost and Moore, 2011; Arnsten et al., 2012; Matsumoto and Takada, 2013; Happel et al., 2014). However, because of the complexity of the mammalian cortex, correlating how animal behavior is changed with DA modulation of specific recurrent networks is difficult. To identify how DA regulates persistent or repetitive behavior in a much simpler animal, we analyzed how DA dampens a recurrently wired sensory-motor network that restricts $C$. elegans male spicule thrusts to the vulva.

During copulation, the vulva mechanochemosensory cues stimulate the PCS sensory-motor neurons, PCA, PCB, and PCC. PCS activity triggers sex-muscle contractions to produce rhythmic spicule thrusts. For simplicity, we focused our attention on two of the three PCS cells. We demonstrated that, not only do the PCA and PCB receive stimulatory signals from the vulva, they reciprocally stimulate each other when DA signaling is absent (Fig. $8 A, B$ ). This recurrent interaction potentially occurs at the initial period of spicule insertion attempts. Recurrent PCS signaling at the beginning of vulva insertion attempts might serve to reinforce the detection of vulval cues. Although the PCS neurons are functionally redundant, each of them might individually contribute to prodding through detection of distinct vulva aspects (Liu and Sternberg, 1995; Garcia et al., 2001; Liu et al., 2011). PCA is the only ciliated PCS neuron (Sulston et al., 1980); thus, similar to other ciliated neurons, it might sense mechanical and/or chemical cues from the vulva. In contrast, the nonciliated PCB and PCC might detect other less-defined anatomical features of the vulval lips. Therefore, reciprocal communication could allow the PCS to integrate these different sensory modalities to sustain spicule thrusts.

Males can spend minutes displaying repetitive spicule thrusts when attempting to breach the hermaphrodite's vulva (Garcia et al., 2001). Because direct chemical and/or mechanical stimulation has been shown to stimulate $C$. elegans sensory neurons for several seconds (Ezcurra et al., 2011; Kato et al., 2014), the recurrency could potentially sustain the PCS activity, so that they continuously stimulate the sex muscles (Fig. $8 B$ ). However, if 
prodding behavior becomes prolonged, we hypothesize that DA secretion from Rn5A, 7A, and 9A neurons tune down the PCS recurrency (Fig. 8D).

When the male first contacts the vulva, his body posture is flushed with the hermaphrodite cuticle. At the vulva, the Rn5A, $7 \mathrm{~A}$, and $9 \mathrm{~A}$ ray neuron activity is initially unchanged compared with when the male was executing backward scanning locomotion. However, after prolonged prodding, the male adopts a ventrally arched posture, which is correlated with increased dopaminergic ray activity (Koo et al., 2011; Correa et al., 2012). We previously shown that direct activation of the PCS network will slowly stimulate the DA rays (Correa et al., 2012); thus, we favor the hypothesis that, during extended intromission attempts, the recurrent PCS activity eventually induces PCA to facilitate DA secretion from ray neurons (Fig. $8 B$ ). The DA then stimulates DOP-2 receptors to attenuate PCS neurons reciprocal signaling. From our genetic screen, we found that one target of D2-like signaling in the male tail is the UNC-7 (L) gap-junction subunit. The isolated A59T missense mutation affects the first exon of the UNC-7(L) isoform, potentially leaving UNC-7(S) intact to permeate ion passage into other cells. UNC-7(S) might also transduce DA signals in other circuits, but our expression pattern suggests that UNC-7 (L) is the predominant effector of DOP-2 for the male copulatory cells. Our data indicated that, during prolonged prodding, D2-like signaling via UNC-7(L) regulation can direct attenuation of $\mathrm{PCB}$ through one of its electrical partners, HOA (Figs. 1, 8D).

Although the PCS acts in conjunction with the hook neurons, $\mathrm{HOA}$ and $\mathrm{HOB}$, to initiate intromission behavior at the vulva, the two sensilla might also act together to terminate futile insertion attempts. The glutamatergic PCA makes chemical synapses to HOA, which expresses the glutamate-gated chloride channel AVR-14, in addition to DOP-2 and UNC-7. During prolonged insertion attempts, persistent PCS signaling could cause the glutamatergic PCA neurons to induce hyperpolarizing chloride influx into HOA, which can then be relayed into PCB through UNC-7 gap-junctions. Our experimental observations indicate that, as a consequence, the DOP-2-dependent HOA-PCB interaction likely dampens PCB's ability to be stimulated by PCA (Fig. $8 C, D)$ and possibly also by its other recurrent partner PCC. The recurring amplification between the cells should be reduced, causing the primary stimulation of the circuit to shift back to the sensilla's sensory input. If the male's sensory apparatus becomes desensitized by prolonged contact with the vulva, then the reduction in PCS recurrency might allow him to move off the vulva.

The regulation of the male intromission circuit displays some parallels and differences with how DA-mediated gap-junction modulation alters circuit interactions in other model systems, such as the vertebrate retina (Ribelayga et al., 2002, 2008; Kothmann et al., 2009). The specialized rods and cones sensory neurons respond to low versus high light conditions, respectively. Studies suggest that, in addition to sensory input, DA signaling can mediate preferential cone activity during bright daylight (Ribelayga et al., 2002, 2008). During the day, release of retinal circadian-regulated DA triggers D2-like signaling in rods and cones to dampen their electrical coupling, thus leaving cones solely responsive to light stimuli (Ribelayga et al., 2002, 2008). In contrast, during the night, decreased DA release allows robust rods-to-cones coupling (Ribelayga et al., 2008). Although D2like signaling dampens cell-cell interactions in both the worm and vertebrate contexts, retinal D2-like signaling diminishes gapjunction-mediated stimulatory cation transport between rods and cone cells (Ribelayga et al., 2008), whereas in the worm,
DOP-2 upregulates UNC-7 to introduce inhibitory signals, possibly chloride, from HOA to PCB.

In the $C$. elegans locomotor circuit, UNC-7 and UNC-9 form heterotypic gap-junctions between cells. When UNC-7 and UNC-9 are individually expressed in the Xenopus oocytes, they can form both heterotypic and homotypic gap-junctions; interestingly, the heterotypic, but not homotypic, gap-junctions show rectifying properties (Starich et al., 2009; Kawano et al., 2011). In previous work, we reported that UNC-9 is also expressed in PCB (Liu et al., 2011). Thus, we do not rule out the possibility that, between HOA and PCB, UNC-7 may form both heterotypic and homotypic gap-junctions. If both types of gap-junctions exist between PCB and HOA, then ion flow could be symmetric or asymmetric, depending on which type of junction is open via upstream neuromodulatory signaling. This type of regulation has been demonstrated in the auditory eight nerve neuron and Mauthner cell interneuron of the auditory vertebrate fish circuit. Between these cells, different connexon subunits make up heterotypic gapjunctions (Korn and Faber, 2005; Rash et al., 2013). Because each subunit has slightly different gating properties, ion passage is heightened from the Mauthner cell onto the eight nerves, resulting in asymmetric ionic distribution (Rash et al., 2013). Moreover, D1-like signaling in the Mauthner cell (Cachope et al., 2007) might contribute to specific heterotypic gap-junction subunit modification and, therefore, directional gating enhancement.

Gap-junction modulation via DA in the vertebrate visual, auditory, and worm copulatory circuits may also provide insight on how firing patterns are regulated in the complex mammalian cortical RNN neurons. In the cortex, GABAergic interneurons contain reciprocal inhibitory chemical connections and are also gap-junction with each other (Galarreta and Hestrin, 1999, 2001). When these cells receive stimulatory glutamate signals from upstream sensory systems, their inhibitory recurrency, in conjunction with gap-junctions, produce amplified oscillatory outputs that drive $30-80 \mathrm{~Hz}$ activity patterns in other cortical neurons. When an animal is conducting cognitive tasks, these cortical oscillations are thought to facilitate the generation of coherent environmental representations, through coordinating parallel sensory CNS processing regions (McBain and Fisahn, 2001). Because these GABAergic neurons also express D2-like receptors, the gap-junctions that contribute to their synchronous oscillations could also be regulated by DA (Galarreta and Hestrin, 2001). This idea is consistent with various studies showing that compulsive and schizophrenic individuals display abnormal DA signaling and oscillatory cortical patterns (Spencer et al., 2004; Wang et al., 2004; Furth et al., 2013; De Koning et al., 2014; Su et al., 2014).

\section{References}

Allen AT, Maher KN, Wani KA, Betts KE, Chase DL (2011) Coexpressed D1- and D2-like dopamine receptors antagonistically modulate acetylcholine release in Caenorhabditis elegans. Genetics 188:579-590. CrossRef Medline

Altun ZF, Chen B, Wang ZW, Hall DH (2009) High resolution map of Caenorhabditis elegans gap-junction proteins. Dev Dyn 238:1936-1950. CrossRef Medline

Arnsten AF, Wang MJ, Paspalas CD (2012) Neuromodulation of thought: flexibilities and vulnerabilities in prefrontal cortical network synapses. Neuron 76:223-239. CrossRef Medline

Barnes TM, Hekimi S (1997) The Caenorhabditis elegans avermectin resistance and anesthetic response gene $u n c-9$ encodes a member of a protein family implicated in electrical coupling of excitable cells. J Neurochem 69:2251-2260. CrossRef Medline

Barr MM, Sternberg PW (1999) A polycystic kidney-disease gene homo- 
logue required for male mating behaviour in C. elegans. Nature 401:386389. CrossRef Medline

Boswell MV, Morgan PG, Sedensky MM (1990) Interaction of GABA and volatile anesthetics in the nematode Caenorhabditis elegans. FASEB J 4:2506-2510. Medline

Brenner S (1974) The genetics of Caenorhabditis elegans. Genetics 77:71-94. Medline

Brozoski TJ, Brown RM, Rosvold HE, Goldman PS (1979) Cognitive deficit caused by regional depletion of dopamine in prefrontal cortex of rhesus monkey. Science 205:929-932. CrossRef Medline

Cachope R, Mackie K, Triller A, O'Brien J, Pereda AE (2007) Potentiation of electrical and chemical synaptic transmission mediated by endocannabinoids. Neuron 56:1034-1047. CrossRef Medline

Chase DL, Pepper JS, Koelle MR (2004) Mechanism of extrasynaptic dopamine signaling in Caenorhabditis elegans. Nat Neurosci 7:1096-1103. CrossRef Medline

Chen TW, Wardill TJ, Sun Y, Pulver SR, Renninger SL, Baohan A, Schreiter ER, Kerr RA, Orger MB, Jayaraman V, Looger LL, Svoboda K, Kim DS (2013) Ultrasensitive fluorescent proteins for imaging neuronal activity. Nature 499:295-300. CrossRef Medline

Correa P, LeBoeuf B, García LR (2012) C. elegans dopaminergic D2-like receptors delimit recurrent cholinergic-mediated motor programs during a goal-oriented behavior. PLoS Genet 8:e1003015. CrossRef Medline

Cully DF, Vassilatis DK, Liu KK, Paress PS, Van der Ploeg LH, Schaeffer JM, Arena JP (1994) Cloning of an avermectin-sensitive glutamate-gated chloride channel from Caenorhabditis elegans. Nature 371:707-711. CrossRef Medline

De Koning MB, Bloemen OJ, Van Duin ED, Booij J, Abel KM, De Haan L, Linszen DH, Van Amelsvoort TA (2014) Pre-pulse inhibition and striatal dopamine in subjects at an ultra-high risk for psychosis. J Psychopharmacol 28:553-560. CrossRef Medline

Dent JA, Davis MW, Avery L (1997) avr-15 encodes a chloride channel subunit that mediates inhibitory glutamatergic neurotransmission and ivermectin sensitivity in Caenorhabditis elegans. EMBO J 16:5867-5879. CrossRef Medline

Dent JA, Smith MM, Vassilatis DK, Avery L (2000) The genetics of ivermectin resistance in Caenorhabditis elegans. Proc Natl Acad Sci U S A 97: 2674-2679. CrossRef Medline

Destexhe A, Contreras D (2006) Neuronal computations with stochastic network states. Science 314:85-90. CrossRef Medline

Edwards SL, Charlie NK, Milfort MC, Brown BS, Gravlin CN, Knecht JE, Miller KG (2008) A novel molecular solution for ultraviolet light detection in Caenorhabditis elegans. PLoS Biol 6:e198. CrossRef Medline

Ezcurra M, Tanizawa Y, Swoboda P, Schafer WR (2011) Food sensitizes C. elegans avoidance behaviours through acute dopamine signalling. EMBO J 30:1110-1122. CrossRef Medline

Furth KE, Mastwal S, Wang KH, Buonanno A, Vullhorst D (2013) Dopamine, cognitive function, and gamma oscillations: role of $\mathrm{D} 4$ receptors. Front Cell Neurosci 7:102. CrossRef Medline

Galarreta M, Hestrin S (1999) A network of fast-spiking cells in the neocortex connected by electrical synapses. Nature 402:72-75. CrossRef Medline

Galarreta M, Hestrin S (2001) Spike transmission and synchrony detection in networks of GABAergic interneurons. Science 292:2295-2299. CrossRef Medline

Garcia LR, Mehta P, Sternberg PW (2001) Regulation of distinct muscle behaviors controls the $C$. elegans male's copulatory spicules during mating. Cell 107:777-788. CrossRef Medline

Ghosh R, Andersen EC, Shapiro JA, Gerke JP, Kruglyak L (2012) Natural variation in a chloride channel subunit confers avermectin resistance in $C$. elegans. Science 335:574-578. CrossRef Medline

Goldman-Rakic PS, Leranth C, Williams SM, Mons N, Geffard M (1989) Dopamine synaptic complex with pyramidal neurons in primate cerebral cortex. Proc Natl Acad Sci U S A 86:9015-9019. CrossRef Medline

Gruninger TR, Gualberto DG, Garcia LR (2008) Sensory perception of food and insulin-like signals influence seizure susceptibility. PLoS Genet 4:e1000117. CrossRef Medline

Haber SN (2014) The place of dopamine in the cortico-basal ganglia circuit. Neuroscience 282C:248-257. CrossRef Medline

Happel MF, Deliano M, Handschuh J, Ohl FW (2014) Dopaminemodulated recurrent corticoefferent feedback in primary sensory cortex promotes detection of behaviorally relevant stimuli. J Neurosci 34:12341247. CrossRef Medline
Hecht RM, Norman MA, Vu T, Jones W (1996) A novel set of uncoordinated mutants in Caenorhabditis elegans uncovered by cold-sensitive mutations. Genome 39:459-464. CrossRef Medline

Hernando G, Bouzat C (2014) Caenorhabditis elegans neuromuscular junction: GABA receptors and ivermectin action. PLoS One 9:e95072. CrossRef Medline

Hodgkin J, Horvitz HR, Brenner S (1979) Nondisjunction mutants of the nematode Caenorhabditis elegans. Genetics 91:67-94. Medline

Iwasaki K, Staunton J, Saifee O, Nonet M, Thomas JH (1997) aex-3 encodes a novel regulator of presynaptic activity in C. elegans. Neuron 18:613622. CrossRef Medline

Jarrell TA, Wang Y, Bloniarz AE, Brittin CA, Xu M, Thomson JN, Albertson DG, Hall DH, Emmons SW (2012) The connectome of a decisionmaking neural network. Science 337:437-444. CrossRef Medline

Jones AK, Sattelle DB (2008) The cys-loop ligand-gated ion channel gene superfamily of the nematode, Caenorhabditis elegans. Invert Neurosci 8:41-47. CrossRef Medline

Kagawa $\mathrm{H}$, Sugimoto $\mathrm{K}$, Matsumoto $\mathrm{H}$, Inoue T, Imadzu $\mathrm{H}$, Takuwa $\mathrm{K}$, Sakube Y (1995) Genome structure, mapping and expression of the tropomyosin gene tmy-1 of Caenorhabditis elegans. J Mol Biol 251:603-613. CrossRef Medline

Kato S, Xu Y, Cho CE, Abbott LF, Bargmann CI (2014) Temporal responses of $C$. elegans chemosensory neurons are preserved in behavioral dynamics. Neuron 81:616-628. CrossRef Medline

Kawano T, Po MD, Gao S, Leung G, Ryu WS, Zhen M (2011) An imbalancing act: gap-junctions reduce the backward motor circuit activity to bias C. elegans for forward locomotion. Neuron 72:572-586. CrossRef Medline

Kim E, Sun L, Gabel CV, Fang-Yen C (2013) Long-term imaging of Caenorhabditis elegans using nanoparticle-mediated immobilization. PLoS One 8:e53419. CrossRef Medline

Kirkby LA, Feller MB (2013) Intrinsically photosensitive ganglion cells contribute to plasticity in retinal wave circuits. Proc Natl Acad Sci U S A 110:12090-12095. CrossRef Medline

Koo PK, Bian X, Sherlekar AL, Bunkers MR, Lints R (2011) The robustness of Caenorhabditis elegans male mating behavior depends on the distributed properties of ray sensory neurons and their output through core and male-specific targets. J Neurosci 31:7497-7510. CrossRef Medline

Korn H, Faber DS (2005) The Mauthner cell half a century later: a neurobiological model for decision-making? Neuron 47:13-28. CrossRef Medline

Kothmann WW, Massey SC, O’Brien J (2009) Dopamine-stimulated dephosphorylation of connexin 36 mediates AII amacrine cell uncoupling. J Neurosci 29:14903-14911. CrossRef Medline

LeBoeuf B, Gruninger TR, Garcia LR (2007) Food deprivation attenuates seizures through CaMKII and EAG K ${ }^{+}$channels. PLoS Genet 3:16221632. CrossRef Medline

LeBoeuf B, Correa P, Jee C, Garcia LR (2014) Caenorhabditis elegans male sensory-motor neurons and dopaminergic support cells couple ejaculation and post-ejaculatory behaviors. Elife 3 .

Liu KS, Sternberg PW (1995) Sensory regulation of male mating behavior in Caenorhabditis elegans. Neuron 14:79-89. CrossRef Medline

Liu Y, LeBeouf B, Guo X, Correa PA, Gualberto DG, Lints R, Garcia LR (2011) A cholinergic-regulated circuit coordinates the maintenance and bi-stable states of a sensory-motor behavior during Caenorhabditis elegans male copulation. PLoS Genet 7:e1001326. CrossRef Medline

Matsumoto M, Takada M (2013) Distinct representations of cognitive and motivational signals in midbrain dopamine neurons. Neuron 79:10111024. CrossRef Medline

McBain CJ, Fisahn A (2001) Interneurons unbound. Nat Rev Neurosci 2:11-23. CrossRef Medline

Nagel G, Brauner M, Liewald JF, Adeishvili N, Bamberg E, Gottschalk A (2005) Light activation of channelrhodopsin-2 in excitable cells of Caenorhabditis elegans triggers rapid behavioral responses. Curr Biol 15: 2279-2284. CrossRef Medline

Noudoost B, Moore T (2011) Control of visual cortical signals by prefrontal dopamine. Nature 474:372-375. CrossRef Medline

Pereda A, Triller A, Korn H, Faber DS (1992) Dopamine enhances both electrotonic coupling and chemical excitatory postsynaptic potentials at mixed synapses. Proc Natl Acad Sci U S A 89:12088-12092. CrossRef Medline

Rash JE, Curti S, Vanderpool KG, Kamasawa N, Nannapaneni S, PalaciosPrado N, Flores CE, Yasumura T, O’Brien J, Lynn BD, Bukauskas FF, 
Nagy JI, Pereda AE (2013) Molecular and functional asymmetry at a vertebrate electrical synapse. Neuron 79:957-969. CrossRef Medline

Redgrave P, Gurney K (2006) The short-latency dopamine signal: a role in discovering novel actions? Nat Rev Neurosci 7:967-975. CrossRef Medline

Reiner DJ, Weinshenker D, Tian H, Thomas JH, Nishiwaki K, Miwa J, Gruninger T, Leboeuf B, Garcia LR (2006) Behavioral genetics of Caenorhabditis elegans-encoded erg-like $\mathrm{K}(+)$ channel. J Neurogenet 20:41-66. CrossRef Medline

Ribelayga C, Wang Y, Mangel SC (2002) Dopamine mediates circadian clock regulation of rod and cone input to fish retinal horizontal cells. J Physiol 544:801-816. CrossRef Medline

Ribelayga C, Cao Y, Mangel SC (2008) The circadian clock in the retina controls rod-cone coupling. Neuron 59:790-801. CrossRef Medline

Sawin ER, Ranganathan R, Horvitz HR (2000) C. elegans locomotory rate is modulated by the environment through a dopaminergic pathway and by experience through a serotonergic pathway. Neuron 26:619-631. CrossRef Medline

Schnabel H, Schnabel R (1990) An organ-specific differentiation gene, pha-1, from Caenorhabditis elegans. Science 250:686-688. CrossRef Medline

Shao YR, Isett BR, Miyashita T, Chung J, Pourzia O, Gasperini RJ, Feldman DE (2013) Plasticity of recurrent L2/3 Inhibition and gamma oscillations by whisker experience. Neuron 80:210-222. CrossRef Medline

Shepherd GM, Stepanyants A, Bureau I, Chklovskii D, Svoboda K (2005) Geometric and functional organization of cortical circuits. Nat Neurosci 8:782-790. CrossRef Medline

Sherlekar AL, Janssen A, Siehr MS, Koo PK, Caflisch L, Boggess M, Lints R (2013) The C. elegans male exercises directional control during mating through cholinergic regulation of sex-shared command interneurons. PLoS One 8:e60597. CrossRef Medline

Spencer KM, Nestor PG, Perlmutter R, Niznikiewicz MA, Klump MC, Frumin M, Shenton ME, McCarley RW (2004) Neural synchrony indexes disordered perception and cognition in schizophrenia. Proc Natl Acad Sci U S A 101:17288-17293. CrossRef Medline

Starich TA, Herman RK, Shaw JE (1993) Molecular and genetic analysis of unc-7, a Caenorhabditis elegans gene required for coordinated locomotion. Genetics 133:527-541. Medline

Starich TA, Xu J, Skerrett IM, Nicholson BJ, Shaw JE (2009) Interactions between innexins UNC-7 and UNC-9 mediate electrical synapse specificity in the Caenorhabditis elegans locomotory nervous system. Neural Dev 4:16. CrossRef Medline

Su P, Li S, Chen S, Lipina TV, Wang M, Lai TK, Lee FH, Zhang H, Zhai D, Ferguson SS, Nobrega JN, Wong AH, Roder JC, Fletcher PJ, Liu F (2014) A dopamine D2 receptor-DISC1 protein complex may contribute to antipsychotic-like effects. Neuron 84:1302-1316. CrossRef Medline

Sugiura M, Fuke S, Suo S, Sasagawa N, Van Tol HH, Ishiura S (2005) Characterization of a novel D2-like dopamine receptor with a truncated splice variant and a D1-like dopamine receptor unique to invertebrates from Caenorhabditis elegans. J Neurochem 94:1146-1157. CrossRef Medline

Sulston JE, Albertson DG, Thomson JN (1980) The Caenorhabditis elegan male: postembryonic development of nongonadal structures. Dev Biol 78:542-576. CrossRef Medline

Sulston J, Dew M, Brenner S (1975) Dopaminergic neurons in the nematode Caenorhabditis elegans. J Comp Neurol 163:215-226. CrossRef Medline

Vassilatis DK, Arena JP, Plasterk RH, Wilkinson HA, Schaeffer JM, Cully DF, Van der Ploeg LH (1997) Genetic and biochemical evidence for a novel avermectin-sensitive chloride channel in Caenorhabditis elegans: isolation and characterization. J Biol Chem 272:33167-33174. CrossRef Medline

Wang M, Vijayraghavan S, Goldman-Rakic PS (2004) Selective D2 receptor actions on the functional circuitry of working memory. Science 303:853856. CrossRef Medline

Wang Y, Belousov AB (2011) Deletion of neuronal gap-junction protein connexin 36 impairs hippocampal LTP. Neurosci Lett 502:30-32. CrossRef Medline

Yeh E, Kawano T, Ng S, Fetter R, Hung W, Wang Y, Zhen M (2009) Caenorhabditis elegans innexins regulate active zone differentiation. J Neurosci 29:5207-5217. CrossRef Medline

Zaldivar D, Rauch A, Whittingstall K, Logothetis NK, Goense J (2014) Dopamine-induced dissociation of BOLD and neural activity in macaque visual cortex. Curr Biol 24:2805-2811. CrossRef Medline 